\title{
Geospatial Assessment of Agricultural Drought Vulnerability Using Integrated Three-Dimensional Model In The Upper Dwarakeshwar River Basin In West Bengal, India
}

Ujjal Senapati ( $\sim$ senapati.ujjal2012@gmail.com )

Cooch Behar Panchanan Barma University https://orcid.org/0000-0001-6492-0162

Tapan Kumar Das

Cooch Behar College

\section{Research Article}

Keywords: Agricultural drought, Vulnerability, Exposure Index, Sensitivity Index, Adaptive Capacity Index

Posted Date: July 20th, 2021

DOI: https://doi.org/10.21203/rs.3.rs-684936/v1

License: () (i) This work is licensed under a Creative Commons Attribution 4.0 International License. Read Full License 


\section{Abstract}

It is essential to measure the degree of agricultural drought vulnerability in an underdeveloped rain-fed agro-based economy at the local, regional, and national level. Agricultural drought has become a major concern in respect to the global food crisis for investigation and development of a sustainable agricultural system that sustains the food security of a country. In this research, delineation of the agricultural drought vulnerability (ADV) status has been carried out by multidimensional mixed-method index approach using remote sensing and geographic information system. An integrated three-dimensional model has been adopted for this study. The three indices of this model are - Exposure Index (EI), Sensitivity Index (SI) and Adaptive Capacity Index (ACI). Exposure Index has been calculated using NDDI, LULC, ADI, DF, ADD and PI. Sensitivity Index has been calculated using satellitebased remote sensing factor VHI, NDWI, EVI, NDVI, VCl, NDWI, LST, and TCI. The ACl has been formed by combining the Environmental Adaptive Capacity (EAC), Social Adaptive Capacity (SAC) and Economical Adaptive Capacity (EcAC) Index. Each index has been computed by assigning the weights based on their relative importance by using the Analytic Hierarchy process (AHP) approach. Final results were classified into five vulnerability zones, e.g., very low, low, moderate, high, and very high covering an area $362.32 \mathrm{~km}^{2}, 186.68 \mathrm{~km}^{2}, 568.69 \mathrm{~km}^{2}, 547.05 \mathrm{~km}^{2}$ and $266.89 \mathrm{~km}^{2}$ respectively. Finally, results have been validated with long term Aman paddy yield data (2004 to 2014) through the Yield Anomaly Index (YAl).

\section{Introduction}

Drought is a least known complex climatic phenomenon that affects the Earth's environment (Boken et al. 2005; Badamassi et al. 2019; Pandey et al. 2012), that impacts on the economy of people who are directly and indirectly dependents on agriculture and applied activities (Wilhite 2000; Nagarajan 2009). This crisis is gradually increasing due to climate change and global warming (Bates et al. 2008). Recently, population growth and increasing demand of water for domestic use led's toward water shortage for agriculture all over the world (Dembele and Zwart 2016).

West Bengal (WB) is a predominantly agricultural based state of India. Almost 70\% population of WB directly or indirectly depends on agriculture for their livelihood, whereas two-thirds of agricultural land is still dependent on monsoonal rainfall for cultivation (Ghosh 2019). The western parts of WB, mainly Bankura and Purulia districts are prone to this recurring drought phenomenon (Bhunia et al. 2020). So, the agriculture is the worst drought-hit sectors in this region (Palchaudhuri and Biswas 2019). The crop production is disrupted due to insufficient root zone soil moisture availability during the growing period of crops (sowing to maturity). As a result, agricultural drought is one of the serious threats to the agro-based rural economy of WB (Dutta et al. 2015).

Agricultural drought vulnerability is the significant aspects of drought management and monitoring for both short and long-term strategies (Murthy et al. 2015; Karet al. 2018). Drought vulnerability differs from one region to another region due to physioclimatic conditions (Sehgal and Dhakar 2016).

Accurate assessment of agricultural drought vulnerability can reduce the probable threat in agricultural sector. So, vulnerability assessment is essential step to increase economic development and agricultural stability in developing countries like India. There are several authorities like- National Agricultural Drought Assessment and Monitoring System (NADAMS) in collaboration with National Remote Sensing Agency (NRSC), The Mahalanobis National Crop Forecast Centre (MNCFC), Indian Meteorological Department (IMD), and agricultural department of different states are involved to monitor and assessment the drought in India. In recent times, researchers have used various methods to assessment agricultural drought vulnerability and risk delineation, likeImproved Projection Pursuit Model (Pei et al. 2016), Analytical Hierarchy Process (Ekrami et al. 2016; Rahman and Lateh 2016), combination of Analytic Hierarchy process (AHP) and fuzzy comprehensive evaluation (Wijitkosum 2018), Probabilistic Assessment Models (Ramadas and Govindaraju 2015), relationship between time series NDVI and rainfall (Murthy et al. 2010), vegetation condition index (VCl) and the standardized precipitation index (SPI) (Dutta et al. 2013; Dutta et al. 2015). Park et al. (2019) have been used random forest model based on index for drought area prediction. Apart from the aforesaid models, Weighted based (on different factors) method has been used by Kar et al. (2018), Zeng et al. (2019), Kim et al. (2018) to assess agriculture drought vulnerability. GIS-based weighted overlay method has also been used to evaluate drought in Jharkhand (Pandey et al. 2012), Ken River basin (Jain et al. 2014), and Rajasthan (Sehgal and Dhakar 2016) using bio-Ecomonic, climatic and social factors. Sensitivity, exposure, and Adaptive capacity indicators were integrated to assess the agricultural drought 
vulnerability (Murthy et al. 2015a; Murthy et al. 2015b; Weis et al. 2016; Fischer and Frazier 2017; Prabnakorn et al. 2019). Although it is noticeable that there is no well accepted universal method is exist to forecast agricultural drought vulnerability. But, recently remote sensing (RS) and Geographical information system (GIS) based work is playing a significant role to monitor agricultural drought forecasting (Chockalingam et al. 2015; Hundera et al. 2016; Sanchez et al. 2018; Trnka et al. 2020). This paper focuses on multi-dimensional holistic index based approach using RS, GIS and AHP techniques constructing integrated Agricultural Drought Vulnerability Index (ADVI) based on three dimensional models in the upper Dwarakeshwar river basin in West Bengal (India) and also to evaluate the geo-spatial distribution of agricultural drought.

\section{Study area:}

The upper Dwarakeshwar river basin is situated in the eastern lower part of Chota-Nagpur plateau. The basin comprises a bordering area amidst the two districts (e.g., Bankura and Purulia) which are located in the western province of West Bengal, a state in the eastern part of India. The study area lies between $23^{\circ} 08^{\prime} 58.80 \otimes N$ to $23^{\circ} 31^{\prime} 55.88 \otimes N$ and $86^{\circ} 30^{\prime} 52.43 \otimes \mathrm{E}$ to $87^{\circ} 09$ ' $13.34 \mathrm{E}$, covering an area of $1934 \mathrm{sq} \mathrm{km}$. The basin is situated in the middle part of the Puruliya district comprising the Kashipur, Puncha, Hura, Santuri, Para and Raghunathpur-I blocks while in Bankura district it covers the Chhatna, Bankura-l, Bankura-II, Indpur, Onda, Gangajalghati and Saltora blocks (Figure.1). The region receives an average annual rainfall about $1528.37 \mathrm{~mm}$. It is also noted that about $80 \%$ of the rainfall is recorded due to the influence of south-west monsoon (June to October). Here, Kharif is the main cropping season and the major crops are Aman paddy that is normally cultivated during this time. On other side, Wheat, mustard, till and potato are cultivated in winter season as robi crops.

\section{Materials And Methods}

\section{Data used}

To perform work, different types of data (e.g., remote sensing, metrological, morphological, soil, drainage, groundwater, and socioeconomic status) have been used in calculation of the aforesaid three composite indexes. The detail description of data source and their specifications for Agricultural Drought Vulnerability Index (ADVI) are given below in Table 1.

Table 1 Details of Data sources used for thematic layers preparation 


\begin{tabular}{|c|c|}
\hline Data & Source \\
\hline \multicolumn{2}{|l|}{ Exposure index } \\
\hline $\begin{array}{l}\text { LANDSAT } 8 \text { (spatial resolution } 30 \text { m) } \\
\text { (LC08_L1TP_139044_20181220_20181227_01_T1) }\end{array}$ & $\begin{array}{l}\text { USGS earth explorer } \\
\text { (https://earthexplorer.usgs.gov/) }\end{array}$ \\
\hline Rainfall data (Daily basis) from 2003 to 2013 & $\begin{array}{l}\text { Climate forecast System Reanalysis } \\
\text { (https://globalweather.tamu.edu/) }\end{array}$ \\
\hline \multicolumn{2}{|l|}{ Sensitivity index } \\
\hline LANDSAT/LC08/C01/T1_8DAY_NDVI (30 meters spatial resolution) in 2016 & $\begin{array}{l}\text { Google earth engine } \\
\text { (https://code.earthengine.google.com/) }\end{array}$ \\
\hline $\begin{array}{l}\text { LANDSAT/LC08/C01/T1_8DAY_NDWI (30 meters spatial resolution) in } \\
2016\end{array}$ & $\begin{array}{l}\text { Google earth engine } \\
\text { (https://code.earthengine.google.com/) }\end{array}$ \\
\hline LANDSAT/LC08/C01/T1_8DAY_EVI(30 meters spatial resolution) in 2016 & $\begin{array}{l}\text { Google earth engine } \\
\text { (https://code.earthengine.google.com/) }\end{array}$ \\
\hline LST (MODIS/006/MOD11A1) 1000 meters spatial resolution in 2016 & $\begin{array}{l}\text { Google earth engine } \\
\text { (https://code.earthengine.google.com/) }\end{array}$ \\
\hline \multicolumn{2}{|l|}{ Adaptive capacity index } \\
\hline Ground water data & $\begin{array}{l}\text { Central Ground Water Board (CGWB), India. } \\
\text { (http://cgwb.gov.in/) }\end{array}$ \\
\hline $\begin{array}{l}\text { SRTM DEM(n23_e086_1arc_v3,n23_e087_1arc_v3)spatial resolution } 30 \\
\text { meters }\end{array}$ & $\begin{array}{l}\text { USGS earth explorer } \\
\text { (https://earthexplorer.usgs.gov/) }\end{array}$ \\
\hline Rainfall data & $\begin{array}{l}\text { Indian Meteorological Department (IMD),Pune } \\
\text { (http://dsp.imdpune.gov.in/) }\end{array}$ \\
\hline Soil depth, texture, and Drainage (Scale $-1: 50,000)$ & $\begin{array}{l}\text { National Bureau of Soil Survey and Land Use } \\
\text { planning (NBSS\&LUP) }\end{array}$ \\
\hline Aquifer systems of India (Scale $-1: 50,000)$ & $\begin{array}{l}\text { Central Ground water Board, Ministry of water } \\
\text { Resources, Government of India. }\end{array}$ \\
\hline Population, no of agricultural labour and farmer, Literacy relates & Census of India, 2011 \\
\hline $\begin{array}{l}\text { Health data, Old-aged Persons, Road(KM), drinking water facility, fertilizer } \\
\text { depots, Seed stores, production, livestock, Pisiculture }\end{array}$ & $\begin{array}{l}\text { District Statistical handbook, Bankura and } \\
\text { Puruliya, in } 2014\end{array}$ \\
\hline Irrigated area & $\begin{array}{l}\text { Irrigation and Waterways Directorate, Govt of } \\
\text { W.B, }\end{array}$ \\
\hline
\end{tabular}

\section{Methodology}

Three principal composite indexes these are Sensitivity Index (SI), Exposure Index (EI), and Adaptive Capacity Index (ACI) have been taken for vulnerability assessment. Each composite index has been generated using AHP technique which has the ability to explain complex problems and make the decision where multiple factors are employed. Factors of each individual composite index (e.g., SI, El and ACI), have been shown in Table 2. Them, multi-dimensional index-based integrated ADVI has also been measured using this formula $[A D V I=(E I+S I)-A C l]$. The details methodology of this research is shown using flow chart (Pic. 2).

Table 2 Selected parameters for agricultural drought vulnerability assessment in the upper Dwarakeshwar river basin. 


\begin{tabular}{|ll}
\hline $\begin{array}{l}\text { Vulnerability } \\
\text { component }\end{array}$ & $\begin{array}{l}\text { Thematic layers } \\
\text { Sensitivity }\end{array}$ \\
$\begin{array}{ll}\text { (1) Vegetation Health Index (VHI), (2) Normalized Difference Water Index (NDWI), (3) Enhanced Vegetation } \\
\text { Index (EVI), (4) Normalized Difference Vegetation Index (NDVI), (5) Vegetation Condition Index (VCI), (6) } \\
\text { Land Surface Temperature (LST), and (7) Temperature Condition Index (TCI) }\end{array}$ \\
$\begin{array}{ll}\text { Exposure } & \text { (1) Normalized Difference Drought Index (NDDI), (2) Landuse and Landcover (LULC), (3) Average Drought } \\
\text { Intensity (ADI), (4) Drought Frequency (DF), (5) Average Drought Duration (ADD), and (6) Peak Intensity (PI) }\end{array}$ \\
$\begin{array}{ll}\text { Environmental } \\
\text { adaptive } \\
\text { capacity }\end{array}$ & $\begin{array}{l}\text { (1) Average Groundwater Depth (AGD), (2) Rainfall (RA), (3) Drainage Density (DD), (4) Drainage Buffer } \\
\text { Soil Texture (ST), (9) Soil Depth (SDpt), (10) Relative Relief (RR), (11) Elevation (EL), (12) Slope (SL) }\end{array}$ \\
$\begin{array}{l}\text { Economic } \\
\text { adaptive } \\
\text { capacity }\end{array}$ & $\begin{array}{l}\text { (1) Irrigation Area Density (IAD), (2) Crop Production Density (CPD), (3) Agricultural Area Density (AAD), (4) } \\
\text { Density (RD), (8) Ratio of Seed Stores (RSS), (9) Ratio of Fertilizer Depots (RFD) }\end{array}$ \\
$\begin{array}{l}\text { Social } \\
\text { adaptive } \\
\text { capacity }\end{array}$ & $\begin{array}{l}\text { (1) Rural Education (RE), (2) Agricultural Labor Density (ALD), (3) Farmer Density (FD), (4) Population } \\
\text { Density (PD) (5) Old-Age Dependency Population (ODP), (6) and Rural Health Facility (RHF), }\end{array}$ \\
\hline
\end{tabular}

\section{AHP technique}

The Analytical Hierarchy Process (AHP) is a semi-quantitative comprehensive technique that fulfills the objective (Quantitative) and subjective (qualitative) aspect (Sener et al. 2011). It is a Multi Criteria Decision Analysis (MCDA) approach which has been used to judgment of final outcome through the assigned weights of the parameters with the pair-wise comparison matrix (Bera et al. 2019).

Each individual composite index was developed by following formula:

$\mathrm{nCI}=\sum_{i=1}^{n} \mathrm{Wa} \times \mathrm{Ra}$

Where, $\mathrm{nCl}$ is the three composite index of vulnerability, Wa is the each factor assigned of weight, and $\mathrm{Ra}$ is the Relative rating weights of the pair-wise comparison values under a classified factors.

AHP technique is build up by two leading segments. The primary segment is the prime scheme of Normalized pair-wise comparison matrix and was calculated by the weights for each factor. The secondary segment is calculated by the relative rating weights of all the factors into sub-classes by using pair-wise comparison matrix of each factor. To create a matrix of pair-wise comparison, each criterion is assigned against the other criterion by allocating a relative rank on Satty's scale (Saaty 1980), between 1 (minimum significant) to 9 (maximum significant) (Table 3). The relative scales of all these factors are given based on different criteria, relative influences, preferences and importance etc. In the Pair-based comparison matrix, each parameter in the row follows the opposite value and its significance with the other parameters. Weights for every factor ware obtained from pairwise comparison matrix by normalizing the values and this was determined by dividing each cell with corresponding sum of the column and then averaging the rows of each criterion. The general pair-wise comparison matrix P1 is constructed as follows,

$P 1=\begin{array}{ccc}1 & 2 & 3 \ldots . . n \\ 1 / 2 & 1 & 2 \ldots . . n \\ 1 / 3 & 1 / 2 & 1 \ldots . . n\end{array}$

At last, the consistency of the Pair-based comparison matrix is assessed by Consistency Ratio (CR). CR is calculated by the following equation:

Consistency Ratio $(\mathrm{CR})=\mathrm{Cl} / \mathrm{RI}$

Where,

$\mathrm{Cl}=$ Consistency Index and

Page 5/33 
$\mathrm{RI}=$ Random Index

If, CR is less than or equal to 0.1 , the comparison matrix is considered as consistent, else it will be corrected.

The Random Index (RI) value obtained from the Satty's standard RI table, which is shown in Table 4. The Consistency Index (Cl) is applied and it is calculated using the following equation-

Consistency index $(\mathrm{Cl})=((\gamma \max -n) /(n-1))$

Where,

$\lambda \max =$ the principle Eigen value of matrix.

$\mathrm{n}=$ Number of parameters used in the analysis.

Table 3 The satty's 9-point relative scale

\begin{tabular}{|llllllllll|}
\hline Scale's & 1 & 2 & 3 & 4 & 5 & 6 & 7 & 8 & 9 \\
$\begin{array}{l}\text { Degree of } \\
\text { importance }\end{array}$ & Equal & Weak & Slight & Moderate & Quite & $\begin{array}{l}\text { Very } \\
\text { strong }\end{array}$ & Extreme & $\begin{array}{l}\text { very strong } \\
\text { Extreme }\end{array}$ & Absolute \\
\hline
\end{tabular}

Table 4 Random Index (RI) value (Saaty 1990)

\begin{tabular}{|lllllllllllll|}
\hline $\mathrm{n}$ & 1 & 2 & 3 & 4 & 5 & 6 & 7 & 8 & 9 & 10 & 11 & 12 \\
\hline $\mathrm{RI}$ & 0.0 & 0.0 & 0.58 & 0.90 & 1.12 & 1.24 & 1.32 & 1.41 & 1.45 & 1.49 & 1.51 & 1.48 \\
\hline
\end{tabular}

Here, all the index parameters of normalized weights value shown in Table 5 and sub-classes weights value of all the parameters shown in Table 6 \& 7.

Table 5 Normalized weights of Exposure index (EI), Sensitivity index (SI) Environmental Adaptive Capacity (EAC) index, Economic Adaptive Capacity (EcAC) and Social Adaptive Capacity (SAC)

\begin{tabular}{|c|c|c|c|c|c|c|c|c|c|}
\hline Parameter of El & weight & $\begin{array}{l}\text { Parameter } \\
\text { of SI }\end{array}$ & weights & $\begin{array}{l}\text { Parameter } \\
\text { of EAC }\end{array}$ & weights & $\begin{array}{l}\text { Parameter } \\
\text { of SAC }\end{array}$ & weights & $\begin{array}{l}\text { Parameter } \\
\text { of EcAC }\end{array}$ & weights \\
\hline NDDI & 0.379 & VHI & 0.338 & AGD & 0.237 & RE & 0.379 & $I A D$ & 0.306 \\
\hline LULC & 0.248 & NDWI & 0.224 & RA & 0.199 & ALD & 0.248 & CPD & 0.218 \\
\hline$A D I$ & 0.160 & EVI & 0.143 & $\mathrm{DD}$ & 0.123 & FD & 0.160 & $A A D$ & 0.154 \\
\hline ADD & 0.102 & NDVI & 0.112 & DB & 0.122 & PD & 0.102 & LD & 0.108 \\
\hline DF & 0.065 & $\mathrm{VCl}$ & 0.079 & SD & 0.089 & ODP & 0.065 & DWF & 0.076 \\
\hline \multirow[t]{7}{*}{$\mathrm{PI}$} & 0.043 & LST & 0.056 & NDVI & 0.065 & RHF & 0.043 & PAD & 0.053 \\
\hline & & $\mathrm{TCl}$ & 0.045 & AM & 0.048 & & & $\mathrm{RD}$ & 0.037 \\
\hline & & & & ST & 0.033 & & & RSS & 0.025 \\
\hline & & & & SDpt & 0.031 & & & RFD & 0.018 \\
\hline & & & & $\mathrm{RR}$ & 0.021 & & & & \\
\hline & & & & EL & 0.012 & & & & \\
\hline & & & & SL & 0.012 & & & & \\
\hline
\end{tabular}

Table 6 Normalize assign weight values for all subcategories of Exposure Index (EI) and Sensitivity Index (SI). 


\begin{tabular}{|c|c|c|c|c|c|}
\hline Parameter & $\begin{array}{l}\text { Sub-classes of Exposure Index } \\
\text { (El) }\end{array}$ & weights & Parameter & $\begin{array}{l}\text { Sub-classes of Sensitivity Index } \\
\text { (SI) }\end{array}$ & weights \\
\hline \multirow[t]{5}{*}{ NDDI } & $0.61-1$ & 0.419 & \multirow[t]{5}{*}{ VHI } & $76-93$ & 0.344 \\
\hline & $0.41-0.60$ & 0.263 & & $61-75$ & 0.344 \\
\hline & $0.31-0.40$ & 0.160 & & $56-60$ & 0.177 \\
\hline & $0.01-0.3$ & 0.097 & & $46-55$ & 0.088 \\
\hline & $-1-0$ & 0.062 & & $45-11$ & 0.047 \\
\hline \multirow[t]{5}{*}{ LULC } & Agricultural land & 0.427 & \multirow[t]{5}{*}{ NDWI } & $0.21-04$ & 0.386 \\
\hline & Natural vegetation & 0.260 & & $0.16-0,2$ & 0.246 \\
\hline & Fallow land & 0.158 & & $0.11-0.15$ & 0.173 \\
\hline & Settlement & 0.096 & & $0.01-0.1$ & 0.120 \\
\hline & Water Body & 0.059 & & -0.1 & 0.075 \\
\hline \multirow[t]{5}{*}{ ADI } & -1.37 to -1.35 & 0.471 & \multirow[t]{5}{*}{ EVI } & $0.41-0.57$ & 0.471 \\
\hline & -.35 to -1.34 & 0.268 & & $0.31-0.4$ & 0.268 \\
\hline & -1.33 to -1.35 & 0.143 & & $0.21-0.3$ & 0.143 \\
\hline & -1.31 to -1.30 & 0.075 & & $0.11-0.2$ & 0.075 \\
\hline & -1.29 to -1.28 & 0.044 & & -0.13 & 0.044 \\
\hline \multirow[t]{5}{*}{$\mathrm{DD}$} & $2.71-2.87$ & 0.445 & \multirow[t]{5}{*}{ NDVI } & $0.301-0.4$ & 0.492 \\
\hline & $2.53-2.7$ & 0.262 & & $0.201-0.3$ & 0.270 \\
\hline & $2.36-2.52$ & 0.153 & & $0.101-0.2$ & 0.135 \\
\hline & $2.18-2.35$ & 0.088 & & $0.001-0.1$ & 0.065 \\
\hline & $2-2.17$ & 0.052 & & $-0-0.0797$ & 0.037 \\
\hline \multirow[t]{5}{*}{ AF } & $13.69-14.9$ & 0.419 & \multirow[t]{5}{*}{$\mathrm{VCl}$} & $81-100$ & 0.419 \\
\hline & 13.39-13.68 & 0.263 & & $61-80$ & 0.263 \\
\hline & 13.13-13.38 & 0.160 & & $41-60$ & 0.160 \\
\hline & $12.86-13.12$ & 0.097 & & $21-40$ & 0.097 \\
\hline & $12.50-12.85$ & 0.062 & & $0-20$ & 0.062 \\
\hline \multirow[t]{4}{*}{$\mathrm{PI}$} & -2.72 to -2.56 & 0.419 & \multirow[t]{4}{*}{ LST } & $30.28-31.86$ & 0.365 \\
\hline & -2.55 to -2.39 & 0.263 & & $31.87-32.29$ & 0.275 \\
\hline & -2.38 to -2.22 & 0.160 & & $32.3-32.7$ & 0.191 \\
\hline & -2.21 to -2.06 & 0.097 & & $32.71-33.28$ & 0.106 \\
\hline
\end{tabular}

Page 7/33 


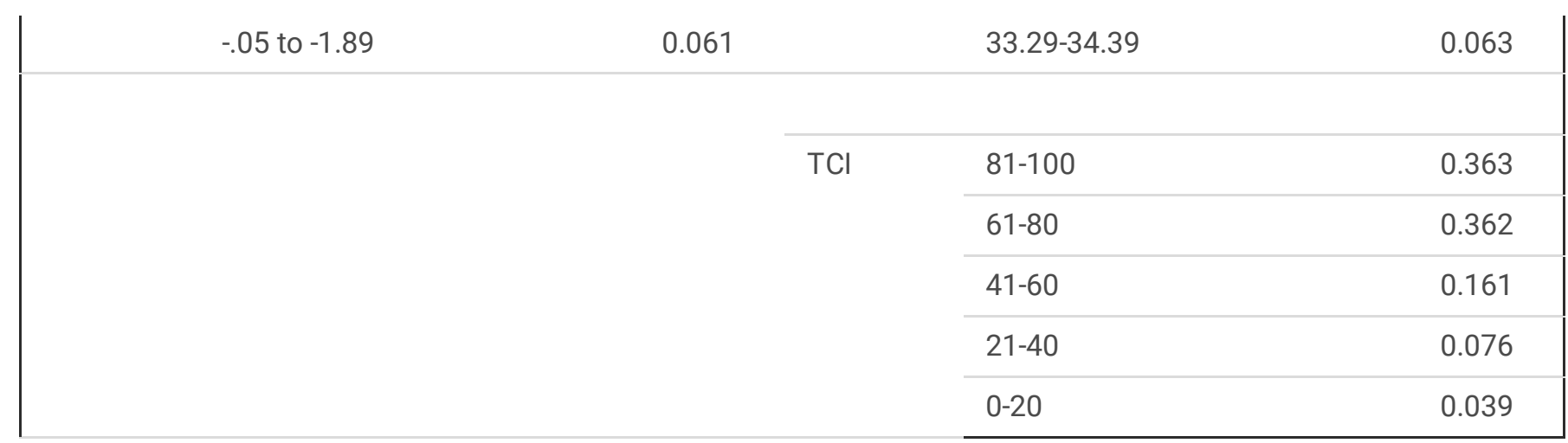

Table 7 Normalize assign weight values for all subcategories of environmental adaptive capacity (EAC) index, Economic Adaptive Capacity (EcAC) and Social Adaptive Capacity (SAC) 


\begin{tabular}{|c|c|c|c|c|c|c|c|c|}
\hline $\begin{array}{l}\text { Parameter of } \\
\text { EAC }\end{array}$ & Sub-classes & weights & $\begin{array}{l}\text { Parameter of } \\
\text { SAC }\end{array}$ & $\begin{array}{l}\text { Sub- } \\
\text { classes }\end{array}$ & weights & $\begin{array}{l}\text { Parameter } \\
\text { of EcAC }\end{array}$ & $\begin{array}{l}\text { Sub- } \\
\text { classes }\end{array}$ & weights \\
\hline \multirow{5}{*}{$\begin{array}{l}\text { Average } \\
\text { groundwater } \\
\text { depth }\end{array}$} & $2.13-3.92$ & 0.445 & \multirow{5}{*}{$\begin{array}{l}\text { Rural } \\
\text { Education }\end{array}$} & $0.8-1$ & 0.445 & \multirow{5}{*}{$\begin{array}{l}\text { Irrigation area } \\
\text { density }\end{array}$} & $0.8-1$ & 0.513 \\
\hline & $3.93-4.45$ & 0.262 & & $0.6-0.8$ & 0.262 & & $0.6-0.8$ & 0.262 \\
\hline & $4.46-4.97$ & 0.153 & & $\begin{array}{l}0.4- \\
0.6\end{array}$ & 0.153 & & $\begin{array}{l}0.4- \\
0.6\end{array}$ & 0.129 \\
\hline & $4.98-6.05$ & 0.089 & & $\begin{array}{l}0.2- \\
0.4\end{array}$ & 0.089 & & $\begin{array}{l}0.2- \\
0.4\end{array}$ & 0.063 \\
\hline & $6.05-8.82$ & 0.052 & & $0-0.2$ & 0.052 & & $0-0.2$ & 0.033 \\
\hline \multirow[t]{5}{*}{ Rainfall } & $1585-1631$ & 0.418 & \multirow{5}{*}{$\begin{array}{l}\text { Agricultural } \\
\text { Labour } \\
\text { Density }\end{array}$} & $0.8-1$ & 0.513 & \multirow{5}{*}{$\begin{array}{l}\text { Crop } \\
\text { production } \\
\text { density }\end{array}$} & $0.8-1$ & 0.471 \\
\hline & $1540-1584$ & 0.263 & & $0.6-0.8$ & 0.262 & & $0.6-0.8$ & 0.268 \\
\hline & $1499-1539$ & 0.160 & & $\begin{array}{l}0.4- \\
0.6\end{array}$ & 0.130 & & $\begin{array}{l}0.4- \\
0.6\end{array}$ & 0.143 \\
\hline & $1454-1498$ & 0.097 & & $\begin{array}{l}0.2- \\
0.4\end{array}$ & 0.063 & & $\begin{array}{l}0.2- \\
0.4\end{array}$ & 0.075 \\
\hline & $1398-1453$ & 0.062 & & $0-0.2$ & 0.033 & & $0-0.2$ & 0.044 \\
\hline \multirow{5}{*}{$\begin{array}{l}\text { Drainage } \\
\text { density }\end{array}$} & $2-3.6$ & 0.471 & \multirow{5}{*}{$\begin{array}{l}\text { Farmer } \\
\text { Density }\end{array}$} & $0.8-1$ & 0.471 & \multirow{5}{*}{$\begin{array}{l}\text { Agricultural } \\
\text { area Density }\end{array}$} & $0.8-1$ & 0.419 \\
\hline & $1.5-1.9$ & 0.264 & & $0.6-0.8$ & 0.268 & & $0.6-0.8$ & 0.263 \\
\hline & $1.2-1.4$ & 0.143 & & $\begin{array}{l}0.4- \\
0.6\end{array}$ & 0.143 & & $\begin{array}{l}0.4- \\
0.6\end{array}$ & 0.160 \\
\hline & $0.73-1.1$ & 0.078 & & $\begin{array}{l}0.2- \\
0.4\end{array}$ & 0.075 & & $\begin{array}{l}0.2- \\
0.4\end{array}$ & 0.097 \\
\hline & $0.0021-0.72$ & 0.044 & & $0-0.2$ & 0.043 & & $0-0.2$ & 0.062 \\
\hline \multirow[t]{5}{*}{ Drainage buffer } & 100 & 0.471 & \multirow{5}{*}{$\begin{array}{l}\text { Population } \\
\text { Density }\end{array}$} & $0.8-1$ & 0.419 & \multirow{5}{*}{$\begin{array}{l}\text { Livestock } \\
\text { density }\end{array}$} & $0.8-1$ & 0.470 \\
\hline & 300 & 0.268 & & $0.6-0.8$ & 0.263 & & $0.6-0.8$ & 0.262 \\
\hline & 500 & 0.143 & & $\begin{array}{l}0.4- \\
0.6\end{array}$ & 0.160 & & $\begin{array}{l}0.4- \\
0.6\end{array}$ & 0.144 \\
\hline & 1000 & 0.075 & & $\begin{array}{l}0.2- \\
0.4\end{array}$ & 0.097 & & $\begin{array}{l}0.2- \\
0.4\end{array}$ & 0.079 \\
\hline & 1500 & 0.044 & & $0-0.2$ & 0.062 & & $0-0.2$ & 0.045 \\
\hline \multirow[t]{5}{*}{ Soil Drainage } & Excessive & 0.350 & \multirow{5}{*}{$\begin{array}{l}\text { Dependent } \\
\text { Population }\end{array}$} & $0.8-1$ & 0.470 & \multirow{5}{*}{$\begin{array}{l}\text { Lack of water } \\
\text { facility } \\
\text { mouza }\end{array}$} & $0.8-1$ & 0.445 \\
\hline & $\begin{array}{l}\text { Somewhat } \\
\text { Excessive }\end{array}$ & 0.276 & & $0.6-0.8$ & 0.262 & & $0.6-0.8$ & 0.262 \\
\hline & Well & 0.159 & & $\begin{array}{l}0.4- \\
0.6\end{array}$ & 0.145 & & $\begin{array}{l}0.4- \\
0.6\end{array}$ & 0.152 \\
\hline & Mod. Well & 0.096 & & $\begin{array}{l}0.2- \\
0.4\end{array}$ & 0.079 & & $\begin{array}{l}0.2- \\
0.4\end{array}$ & 0.089 \\
\hline & Imperfect Well & 0.058 & & $0-0.2$ & 0.045 & & $0-0.2$ & 0.052 \\
\hline
\end{tabular}




\begin{tabular}{|c|c|c|c|c|c|c|c|c|}
\hline & Imperfect Mod & \multicolumn{7}{|l|}{0.037} \\
\hline & Imperfect & \multicolumn{7}{|l|}{0.025} \\
\hline \multirow[t]{5}{*}{ NDVI } & -0.134 & 0.513 & \multirow[t]{5}{*}{ Rural Health } & $0.8-1$ & 0.419 & \multirow{5}{*}{$\begin{array}{l}\text { Pisicultural } \\
\text { density }\end{array}$} & $0.8-1$ & 0.470 \\
\hline & $0.201-0.387$ & 0.275 & & $0.6-0.8$ & 0.263 & & $0.6-0.8$ & 0.262 \\
\hline & $0.101-0.2$ & 0.138 & & $\begin{array}{l}0.4- \\
0.6\end{array}$ & 0.160 & & $\begin{array}{l}0.4- \\
0.6\end{array}$ & 0.144 \\
\hline & $0.001-0.1$ & 0.074 & & $\begin{array}{l}0.2- \\
0.4\end{array}$ & 0.097 & & $\begin{array}{l}0.2- \\
0.4\end{array}$ & 0.079 \\
\hline & & & & $0-0.2$ & 0.062 & & $0-0.2$ & 0.045 \\
\hline \multirow[t]{6}{*}{ Aquifer media } & Older Alluvium & 0.415 & & & & & & \\
\hline & $\begin{array}{l}\text { Older Alluvium, } \\
\text { Sand and Silt }\end{array}$ & 0.255 & & & & \multirow[t]{5}{*}{ Road density } & $0.8-1$ & 0.445 \\
\hline & Laterite & 0.153 & & & & & $0.6-0.8$ & 0.262 \\
\hline & Schist & 0.089 & & & & & $\begin{array}{l}0.4- \\
0.6\end{array}$ & 0.153 \\
\hline & $\begin{array}{l}\text { Banded } \\
\text { Gneissic } \\
\text { Complex }\end{array}$ & 0.054 & & & & & $\begin{array}{l}0.2- \\
0.4\end{array}$ & 0.088 \\
\hline & Basic Intrusives & 0.034 & & & & & $0-0.2$ & 0.052 \\
\hline \multirow[t]{5}{*}{ Soil texture } & $\begin{array}{l}\text { Sandy loamy } \\
\text { group }\end{array}$ & 0.427 & & & & \multirow[t]{5}{*}{$\begin{array}{l}\text { Ration of } \\
\text { seed stores }\end{array}$} & $0.8-1$ & 0.418 \\
\hline & $\begin{array}{l}\text { Sandy clay } \\
\text { group }\end{array}$ & 0.260 & & & & & $0.6-0.8$ & 0.263 \\
\hline & Loamy group & 0.158 & & & & & $\begin{array}{l}0.4- \\
0.6\end{array}$ & 0.160 \\
\hline & Gravelly group & 0.096 & & & & & $\begin{array}{l}0.2- \\
0.4\end{array}$ & 0.097 \\
\hline & Clay group & 0.058 & & & & & $0-0.2$ & 0.061 \\
\hline \multirow[t]{5}{*}{ Soil Depth } & Very Deep & 0.446 & & & & \multirow{5}{*}{$\begin{array}{l}\text { Ratio of } \\
\text { fertilizer } \\
\text { depots }\end{array}$} & $0.8-1$ & 0.419 \\
\hline & Deep & 0.262 & & & & & $0.6-0.8$ & 0.262 \\
\hline & Moderate & 0.152 & & & & & $\begin{array}{l}0.4- \\
0.6\end{array}$ & 0.160 \\
\hline & Shallow & 0.089 & & & & & $\begin{array}{l}0.2- \\
0.4\end{array}$ & 0.097 \\
\hline & Very Shallow & 0.052 & & & & & $0-0.2$ & 0.062 \\
\hline \multirow[t]{3}{*}{ Relative Relief } & $<20$ & 0.471 & & & & & & \\
\hline & $21-25$ & 0.264 & & & & & & \\
\hline & $26-35$ & 0.143 & & & & & & \\
\hline
\end{tabular}

Page 10/33 


\begin{tabular}{|ll|}
\hline \multicolumn{3}{|l|}{$36-45$} & 0.078 \\
\hline Elevation & 0.044 \\
\hline $69-112$ & 0.419 \\
\hline $113-140$ & 0.263 \\
\hline $141-168$ & 0.160 \\
\hline $169-201$ & 0.097 \\
\hline $202-255$ & 0.062 \\
\hline slope & 0.419 \\
\hline $0-1$ & 0.263 \\
\hline $1.1-3$ & 0.160 \\
\hline $3.1-5$ & 0.097 \\
\hline $5.1-7$ & 0.061 \\
\hline$>7$ & \\
\hline
\end{tabular}

\section{Synthesizing the composite index}

\section{Exposure Index (EI)}

Exposure index (EI) is the measurement of the degree of disclosure which received by the drought. Exposure means something particularly embarrassing, damaging or harmful. Here, NDDI, LULC, and Standardized precipitation Index (SPI) based Average Drought Intensity (ADI), Drought Frequency (DF), Drought Duration (DD), and Peak Intensity (PI) indicators are considered (Figure 3 ) to create the exposure index by using GIS based AHP technique. The Parameters of evaluation exposure indexes have been shown in Table 8.

Table 8 Evaluation Exposure Index (EI) for the agricultural drought vulnerability assessment in the upper Dwarakeshwar river basin. 


\begin{tabular}{|c|c|c|c|c|}
\hline $\begin{array}{l}\text { Index } \\
\text { thematic } \\
\text { layer }\end{array}$ & $\begin{array}{l}\text { Formulating } \\
\text { method }\end{array}$ & Description & $\begin{array}{l}\text { Relation with } \\
\text { Exposure }\end{array}$ & Reference \\
\hline NDDI & $\begin{array}{l}\mathrm{NDDI}=((\mathrm{NDVI} \\
-\mathrm{NDWI}) / \\
(\mathrm{NDVI}+ \\
\mathrm{NDWI}))\end{array}$ & $\begin{array}{l}\text { NDDI is a satellite-generated and widely known } \\
\text { frequently used as agricultural drought indicator. } \\
\text { Higher NDDI value indicates higher probability of drought } \\
\text { exposure and vice versa. }\end{array}$ & Positive & $\begin{array}{l}\text { Gu et } \\
\text { al.2007 }\end{array}$ \\
\hline LULC & $\begin{array}{l}\text { Supervised } \\
\text { calcification }\end{array}$ & $\begin{array}{l}\text { LULC is another important factor for assessing exposure } \\
\text { of agricultural drought. Agricultural land, fallow land and } \\
\text { natural vegetation are higher probability of drought } \\
\text { exposure index relatively water bodies and settlement. }\end{array}$ & $\begin{array}{l}\text { Agricultural land } \\
\text { is very high } \\
\text { exposure, and } \\
\text { Water Bodies is } \\
\text { very low. }\end{array}$ & $\begin{array}{l}\text { Biazina and } \\
\text { Sterk } 2013\end{array}$ \\
\hline $\begin{array}{l}\text { Average } \\
\text { Drought } \\
\text { Intensity }\end{array}$ & $\begin{array}{l}\mathrm{ADI}= \\
\left(\mathrm{N}_{1}+\mathrm{N}_{2}+\mathrm{N}_{3} \ldots . .+\right. \\
\mathrm{Nn} / \mathrm{Tm})\end{array}$ & $\begin{array}{l}\text { Higher average drought intensity value indicates higher } \\
\text { probability of drought exposure and vice versa. }\end{array}$ & Positive & $\begin{array}{l}\text { Ghosh. } \\
2019\end{array}$ \\
\hline $\begin{array}{l}\text { Average } \\
\text { Drought } \\
\text { Duration }\end{array}$ & $A D D=\left(D_{S} / D\right)$ & $\begin{array}{l}\text { Higher average drought duration value indicates higher } \\
\text { probability of drought exposure and vice versa. }\end{array}$ & Positive & $\begin{array}{l}\text { Ghosh. } \\
2019\end{array}$ \\
\hline $\begin{array}{l}\text { Drought } \\
\text { Frequency }\end{array}$ & $\begin{array}{l}\mathrm{DF}_{\mathrm{j} .10}=(\mathrm{Nj} / \\
\text { j.n }) \times 100 \%\end{array}$ & $\begin{array}{l}\text { Higher drought frequency value indicates higher } \\
\text { probability of drought exposure and vice versa. }\end{array}$ & Positive & $\begin{array}{l}\text { Wang et } \\
\text { al.2013 }\end{array}$ \\
\hline $\begin{array}{l}\text { Peak } \\
\text { Intensity }\end{array}$ & $\begin{array}{l}\text { Lowest spi } \\
\text { value of } \\
\text { observational } \\
\text { 3-month SPI }\end{array}$ & $\begin{array}{l}\text { Higher peak intensity value indicates higher probability } \\
\text { of drought exposure and vice versa. }\end{array}$ & Positive & $\begin{array}{l}\text { Raha and } \\
\text { Gayen.2020 }\end{array}$ \\
\hline
\end{tabular}

\section{Sensitivity Index (SI)}

Sensitivity in vulnerability assessment is a measure of how much the local climate will change in vulnerability to during drought. Sensitivity is assessment of the susceptibility of moisture stress or water threads for agricultural drought. Vegetation health and freshness, soil moisture, soil temperature, evaporation, and transpiration are all critical factors for assessing agricultural drought sensitivities. Here the Sensitivity index is created using the satellite based remote sensing factor VHI, NDWI, EVI, NDVI, VCl, NDWI, LST, and TCI (Figure 4) and all thematic layers has been prepared and resampling in $30 \mathrm{~m}$ spatial resolution through GIS environment. Parameters of evaluation sensitivity indexes have been shown table 9 . In addition, a combination of eight different indicators has been developed using data from various sensors like Landsat and Modis in 2016 to better understand the agricultural drought sensitivity.

Table 9 Evaluation Sensitivity Index (SI) for the agricultural drought vulnerability assessment in the upper Dwarakeshwar river basin. 


\begin{tabular}{|c|c|c|c|c|}
\hline $\begin{array}{l}\text { Index } \\
\text { thematic } \\
\text { layer }\end{array}$ & $\begin{array}{l}\text { Formulating } \\
\text { method }\end{array}$ & Description & $\begin{array}{l}\text { Relation } \\
\text { With } \\
\text { sensitivity }\end{array}$ & Reference \\
\hline VHI & $\begin{array}{l}\mathrm{VHI}=\mathrm{a} \times \mathrm{VCl} \\
+(1+\mathrm{a}) \times \\
\mathrm{TCl}\end{array}$ & $\begin{array}{l}\mathrm{VHI} \text { is a combination of } \mathrm{VCl} \text { and } \mathrm{TCl} \text { and is a two-dimensional } \\
\text { indicator for assessing the incidence of agricultural drought } \\
\text { temporarily and spatially. Higher } \mathrm{VHI} \text { value represents higher } \\
\text { probability of drought sensitivity. }\end{array}$ & Positive & $\begin{array}{l}\text { sun et al. } \\
2013\end{array}$ \\
\hline NDWI & $\begin{array}{l}\text { NDWI }=((\text { NIR } \\
- \text { SWIR }) /(\text { NIR } \\
+ \text { SWIR }))\end{array}$ & $\begin{array}{l}\text { NDWI is primarily designed to describe the spatial characteristics } \\
\text { of surface open water condition and it used to monitorvegetation } \\
\text { and agricultural droughts. That means Higher NDWI } \\
\text { value indicates lower probability of drought sensitivity and vice } \\
\text { versa. }\end{array}$ & Negative & $\begin{array}{l}\text { Amalo et al. } \\
2018\end{array}$ \\
\hline EVI & $\begin{array}{l}\mathrm{EVI}=2.5 \times( \\
\mathrm{NIR}- \\
\mathrm{RED}) /(\mathrm{NIR}+ \\
6 \times \mathrm{RED}-7.5 \\
\times \mathrm{BLUE}+1)\end{array}$ & $\begin{array}{l}\text { EVI has proven to be an effective way to assess the long-term } \\
\text { trend in vegetation "greening".So, Higher EVI value indicates } \\
\text { higher probability of drought sensitivity and vice versa. }\end{array}$ & Positive & $\begin{array}{l}\text { Zhu et } \\
\text { al.2016 }\end{array}$ \\
\hline NDVI & $\begin{array}{l}\text { NDVI }=(\mathrm{NIR}- \\
\text { RED) / (NIR + } \\
\text { RED) }\end{array}$ & $\begin{array}{l}\text { The seasonal and inter-annual plant growth in a region and } \\
\text { Potential cultivable areas can be identified by NDVI. Higher NDVI } \\
\text { value indicates higher probability of drought sensitivity and vice } \\
\text { versa. }\end{array}$ & Positive & $\begin{array}{l}\text { Bhavani et } \\
\text { al. } 2017\end{array}$ \\
\hline $\mathrm{VCl}$ & $\begin{array}{l}\mathrm{VCl}=((\mathrm{NDVli} \\
-\mathrm{NDVImin}) / \\
(\mathrm{NDVImax}- \\
\text { NDVImin) })^{*} \\
100\end{array}$ & $\begin{array}{l}\mathrm{VCI} \text { derived from NDVI isolates short-term weather-related signals } \\
\text { from long-term environmental factors and is effective in } \\
\text { observing and comparing drought-affected plants across a large } \\
\text { area. So Higher VCI value indicates higher probability of drought } \\
\text { sensitivity and vice versa. }\end{array}$ & Positive & $\begin{array}{l}\text { Palchaudhuri } \\
\text { and Biswas } \\
2019\end{array}$ \\
\hline LST & $\begin{array}{l}\text { LSTC }=(\text { LST } \\
\times 0.02)- \\
273.15\end{array}$ & $\begin{array}{l}\text { LST is a significant parameter for the study of drought and } \\
\text { environment and Higher VCI value indicates higher probability of } \\
\text { drought sensitivity and vice versa. }\end{array}$ & Positive & $\begin{array}{l}\text { Arekhi et al. } \\
2019\end{array}$ \\
\hline $\mathrm{TCl}$ & $\begin{array}{l}\text { TCI }=(\text { LSTli } \\
- \text { LSTmin) / } \\
(\text { LSTmax - } \\
\text { LSTmin }))^{\star} \\
100\end{array}$ & $\begin{array}{l}\text { TCI is involved in the brightness temperature calculated from } \\
\text { LST. Higher VCl value indicates higher probability of drought } \\
\text { sensitivity and vice versa. }\end{array}$ & Positive & $\begin{array}{l}\text { Rojas et al. } \\
2011\end{array}$ \\
\hline
\end{tabular}

\section{Adaptive Capacity Index (ACl)}

Adaptive capacity elaborates the efficiency of acclimatize power. So, Adaptive capacity provides the ability to reconfigure with minimal loss of resilience, environmental, Ecomonic, and human socio-economic system functions. An adaptive capability includes social and technological skills and strategies that allow multiple individuals or groups to adjust the environmental and socio-economic changes. In the context of the food system, adaptive capacity is usually developed or deployed to maintain livelihoods, food production or food access.

In field of drought vulnerability, Adaptive capacity is the inherent strength of the agricultural area to cope with the reduction of the crop productivity and probable loss in the agricultural drought. Here, The ACl is a composite index of three indices, namely Economic Adaptive capacity (EcAC), Environmental Adaptive Capacity (EAC), and Social Adaptive Capacity (SAC).

The SAC and EcAC data was normalized by using the following equations-

If $p$ has positively related to vulnerability then 
$p n=(p a-p \min ) /(p \max -p \min )$

And if $p$ has negatively related to vulnerability then used

$p n=(p m a x-p a) /(p m a x-p m i n)$

Where, $p n$ is normalized parameters, $p \alpha$ is each individual parameter, and $p m a x$ and $p m i n$ respectively represent maximum and minimum value of each parameter.

\section{Environmental Adaptive Capacity (EAC)}

The environmental elements control the amount of potential damage from a potential hazard or disaster and also build the EAC index. To measures EAC index, average groundwater depth, rainfall, drainage density, drainage buffer, soil drainage, NDVI, aquifer media, soil texture, soil depth, relative relief, elevation, slope factors have been used and it has also been attached by AHP technology. All collected data has been thematically mapped in GIS platform at $30 \mathrm{~m}$ spatial resolution (Figure. 5) respectively. Parameters of evaluation EAC has been shown in table 10. Finally, the EAC Index is computed by using the GIS overly analysis method based on the weightage of all the parameters.

Table 10 Evaluation Environmental Adaptive Capacity (EAC) index for the agricultural drought vulnerability assessment in the upper Dwarakeshwar river basin.

\begin{tabular}{|c|c|c|c|}
\hline $\begin{array}{l}\text { Index thematic } \\
\text { layer }\end{array}$ & $\begin{array}{l}\text { Formulating } \\
\text { method }\end{array}$ & Description & $\begin{array}{l}\text { Relation } \\
\text { with EAC }\end{array}$ \\
\hline $\begin{array}{l}\text { Average } \\
\text { groundwater depth }\end{array}$ & $\begin{array}{l}\text { Interpolation (IDW) } \\
\text { method }\end{array}$ & $\begin{array}{l}\text { Higher average groundwater depth higher probability of } \\
\text { drought adaptive capacity. }\end{array}$ & Positive \\
\hline Rainfall & $\begin{array}{l}\text { Interpolation (IDW) } \\
\text { method }\end{array}$ & $\begin{array}{l}\text { Higher Rainfall indicates higher probability of drought adaptive } \\
\text { capacity. }\end{array}$ & Positive \\
\hline Drainage density & $\begin{array}{l}\text { Drainage } \\
\text { length/area }\end{array}$ & $\begin{array}{l}\text { Higher Drainage density indicates higher probability of drought } \\
\text { adaptive capacity. }\end{array}$ & Positive \\
\hline Drainage buffer & Multiple Buffer & $\begin{array}{l}\text { Near the drainage indicates higher probability of drought } \\
\text { adaptive capacity. }\end{array}$ & Positive \\
\hline Soil drainage & Digitalization & $\begin{array}{l}\text { Higher Soil drainage indicates higher probability of drought } \\
\text { adaptive capacity. }\end{array}$ & Positive \\
\hline NDVI & $\begin{array}{l}\text { (Nir - red) } /(\mathrm{Nir}+ \\
\text { red) }\end{array}$ & $\begin{array}{l}\text { Higher value of NDVI indicates higher probability of drought } \\
\text { adaptive capacity. }\end{array}$ & Positive \\
\hline Aquifer media & Digitalization & $\begin{array}{l}\text { Higher replaceable recharge indicates higher probability of } \\
\text { drought adaptive capacity. }\end{array}$ & Positive \\
\hline Soil texture & Digitalization & $\begin{array}{l}\text { Finer the Soil texture indicates higher probability of drought } \\
\text { adaptive capacity. }\end{array}$ & Positive \\
\hline Soil Depth & Digitalization & $\begin{array}{l}\text { Higher Soil Depth indicates higher probability of drought } \\
\text { adaptive capacity. }\end{array}$ & Positive \\
\hline Relative Relief & From DEM & $\begin{array}{l}\text { Higher Relative Relief indicates lower probability of drought } \\
\text { adaptive capacity. }\end{array}$ & Negative \\
\hline Elevation & From DEM & $\begin{array}{l}\text { Higher Elevation indicates lower probability of drought adaptive } \\
\text { capacity. }\end{array}$ & Negative \\
\hline Slope & From DEM & $\begin{array}{l}\text { Higher Slope indicates lower probability of drought adaptive } \\
\text { capacity. }\end{array}$ & Negative \\
\hline
\end{tabular}

Social Adaptive Capacity (SAC) 
The adaptive capacity of a society is created by bringing together the social elements that empower the society from a single disaster. Social adaptive power controls the severity and duration of any kind of catastrophe. Social infrastructures such as education, health, labor force, unity, technology and productivity have the power to control the consequences of any kind of disaster. Here, to diagnose social adaptive capacity, six parameters have been used, such as, agricultural labor density, farmer density, rural literacy rate, old-age dependency population ratio, rural health facility, and population density (Figure.6). Parameters of evaluation SAC has been shown table 11.

The SAC index has been constructed using the GIS overlay method with AHP based assigned weightage on the thematic layers of all the permits based on their normalized value. Thematic layers are farmers, agricultural labor, rural literacy, population density, old age dependency population and health.

Table 11 Evaluation Social Adaptive Capacity (SAC) index for the agricultural drought vulnerability assessment in the upper Dwarakeshwar river basin.

\begin{tabular}{|llll|}
\hline $\begin{array}{l}\text { Index thematic } \\
\text { layer }\end{array}$ & Formulating method & Description & $\begin{array}{c}\text { Relation } \\
\text { with SAC }\end{array}$ \\
\hline Rural education & $\begin{array}{l}\text { Obtained directly from Rural } \\
\text { literacy rate }\end{array}$ & $\begin{array}{l}\text { Higher Rural education indicates higher probability of } \\
\text { drought adaptive capacity. }\end{array}$ & Positive \\
\hline $\begin{array}{l}\text { Agricultural } \\
\text { labour density }\end{array}$ & No of agricultural labour/Area & $\begin{array}{l}\text { Higher Agricultural labour density indicates lower } \\
\text { probability of drought adaptive capacity. }\end{array}$ & Negative \\
\hline Farmer density & No of farmer/Area & $\begin{array}{l}\text { Higher Farmer density indicates lower probability of } \\
\text { drought adaptive capacity. }\end{array}$ & Negative \\
\hline $\begin{array}{l}\text { Population } \\
\text { density }\end{array}$ & Total population/Area & $\begin{array}{l}\text { Higher Population density indicates lower probability of } \\
\text { drought adaptive capacity. }\end{array}$ & Negative \\
$\begin{array}{l}\text { Old age } \\
\text { dependent }\end{array}$ & $\begin{array}{l}\text { No of old age dependency } \\
\text { population/Total Population }\end{array}$ & $\begin{array}{l}\text { Higher Old age dependant Population indicates lower } \\
\text { probability of drought adaptive capacity. }\end{array}$ & Negative \\
\hline $\begin{array}{l}\text { Rural health } \\
\text { (No of bed/population) } \times 100\end{array}$ & $\begin{array}{l}\text { Higher Rural health facility indicates higher probability } \\
\text { of drought adaptive capacity. }\end{array}$ & Positive \\
\hline
\end{tabular}

\section{Economic Adaptive Capacity (EcAC)}

The EcAC Index is formed by the elements that Economically control the ability to adapt any kind of natural phenomena. EcAC index depend on various natural factor which is important index to determine region-based agricultural droughts. These are the road density, drinking water facility, irrigation area, agricultural area, total crop production, ratio of seed stores and livestock for determining the EcAC of agricultural drought in the agro-based upper Dwarakeshwar River Basin which is showing in figure 7. Parameters of evaluation EcAC have been shown in Table. 12.

Table 12 Evaluation Economic Adaptive Capacity (EcAC) index for the agricultural drought vulnerability assessment in the upper Dwarakeshwar river basin. 


\begin{tabular}{|lllll|}
\hline $\begin{array}{l}\text { Index } \\
\text { thematic } \\
\text { layer }\end{array}$ & Formulating method & Description & $\begin{array}{l}\text { Relation } \\
\text { with } \\
\text { EcAC }\end{array}$ \\
\hline $\begin{array}{l}\text { Irrigation area } \\
\text { density }\end{array}$ & $\begin{array}{l}\text { Irrigated area/ total } \\
\text { area }\end{array}$ & $\begin{array}{l}\text { Higher irrigation density indicates a higher adaptive capacity that } \\
\text { means lower vulnerability. }\end{array}$ & Positive \\
$\begin{array}{l}\text { crop } \\
\text { production } \\
\text { density }\end{array}$ & $\begin{array}{l}\text { Total crop } \\
\text { production/ Total } \\
\text { agricultural area }\end{array}$ & $\begin{array}{l}\text { A higher crop production indicates higher probability of drought } \\
\text { adaptive capacity. }\end{array}$ & Positive \\
\hline $\begin{array}{l}\text { Agricultural } \\
\text { area Density }\end{array}$ & $\begin{array}{l}\text { Total agricultural } \\
\text { area/ total area }\end{array}$ & $\begin{array}{l}\text { A higher Agricultural area indicates lower probability of drought } \\
\text { adaptive capacity. }\end{array}$ & Negative \\
\hline $\begin{array}{l}\text { livestock } \\
\text { density }\end{array}$ & $\begin{array}{l}\text { No of livestock/ total } \\
\text { area }\end{array}$ & $\begin{array}{l}\text { Livestock is an alternative source of income. So a higher livestock } \\
\text { density area indicates higher probability of drought adaptive capacity. }\end{array}$ & Positive \\
\hline $\begin{array}{l}\text { Drinking water } \\
\text { facility }\end{array}$ & $\begin{array}{l}\text { Total mouza }- \\
\text { Drinking water } \\
\text { facility mouza }\end{array}$ & $\begin{array}{l}\text { A higher water facility block indicates higher probability of drought } \\
\text { adaptive capacity. }\end{array}$ & Positive \\
\hline $\begin{array}{l}\text { Piscicultural } \\
\text { Area Density }\end{array}$ & $\begin{array}{l}\text { Piscicultural area/ } \\
\text { total area }\end{array}$ & $\begin{array}{l}\text { Pisciculture is an alternative source of income, so higher Piscicultural } \\
\text { density areas indicate higher probability of drought adaptive capacity. }\end{array}$ & Positive \\
\hline Road density & $\begin{array}{l}\text { Length of road / total } \\
\text { area }\end{array}$ & $\begin{array}{l}\text { A higher Road density indicates higher probability of drought adaptive } \\
\text { capacity. }\end{array}$ & Positive \\
\hline $\begin{array}{l}\text { Ration of seed } \\
\text { stores }\end{array}$ & $\begin{array}{l}\text { No of seed stores/ } \\
\text { total area }\end{array}$ & $\begin{array}{l}\text { A higher seed stores density area indicates higher probability of } \\
\text { drought adaptive capacity. }\end{array}$ & $\begin{array}{l}\text { A higher ratio of fertilizer depots indicates higher probability of } \\
\text { drought adaptive capacity. }\end{array}$ \\
\hline $\begin{array}{l}\text { Ratio of } \\
\text { fertilizer } \\
\text { depots }\end{array}$ & $\begin{array}{l}\text { Nopots fertilizer } \\
\text { depotal area }\end{array}$ & Positive \\
\hline
\end{tabular}

\section{Results}

The GIS-based 3 indicators have been used to assess agricultural drought vulnerability. The vulnerability of agricultural drought depends on the regional distribution of these three indicators such as, El, SI and ACl.

The exposure index (EI) of upper Dwarkeswar river basin has been divided into 5 regions such as, very low (El value is $0-20)$, which covering of $52.36 \mathrm{sq} \mathrm{km} \mathrm{(2.71 \% )} \mathrm{area,} \mathrm{low} \mathrm{(21-40)} \mathrm{:} 326.87 \mathrm{sq} \mathrm{km} \mathrm{(16.9 \% )} \mathrm{area,} \mathrm{medium} \mathrm{(41-60)} \mathrm{:} 791.78 \mathrm{sq} \mathrm{km} \mathrm{(40.94 \% )}$ area, high (61-80): $641.58 \mathrm{sq} \mathrm{km} \mathrm{(33.17 \% )} \mathrm{area} \mathrm{and} \mathrm{very} \mathrm{high} \mathrm{(81-100)} \mathrm{:} 121.40 \mathrm{sq} \mathrm{km} \mathrm{(6.28 \% )} \mathrm{area} \mathrm{(Fig.} \mathrm{8).} \mathrm{Deep} \mathrm{green} \mathrm{area} \mathrm{is}$ showing the very low exposure index, covering very small part and scattered portion area of the basin. The light green color indicates the low exposure index region; these are mostly in forest areas. The yellow portion is showing moderate exposure index which is covering mainly north-eastern and central parts of the study area. Brown color portion represent high exposure index, and south western part of the study area shows in very high exposure index which indicate in red color.

The Sensitivity Index (SI) is the second most important indicator of agricultural drought vulnerability. This sensitive index is formed by combining the different satellite-based drought indicators with the help of GIS overlay method. According to Sensitivity Index, the study area is divided into five sub classes, namely 'very high' (81-100), 'high'(61-80), 'medium' (41-60), 'low' (21-40) and 'very poor' (0-20) covering an area of $54.66,257.85,590.52,869.96$ and 171.01 sq km accounting 2.83, 13.33, 30.53, 44.47 and $8.84 \%$, respectively, of the total area (Fig. 9). The Sensitive Index of the Deep Green Region, which is sparsely scattered in the northern and western parts of the study area, is very low, and northern and western region falls within the low sensitivity index. Moderate and high sensitive index has been seen in the middle portion of the study area. Areas with a very high sensitive index are distributed scatterly throughout the study areas which are shown by red color.

Adaptive Capacity Index (ACl) (Fig. 10) these three indices namely EAC, SAC, and EcAC (Fig. 11) have been combined to calculate the Total Adaptive Capacity Index. ACl of the study area is divided into five classes, which are: such as very low (0-20), low (2140), moderate (41-60), high (61-80), and very high (81-100). These classes have covered $334.05 \mathrm{sq} \mathrm{km}$ area $(17.29 \%), 1090.30$ sq km area (56.42 \%), $281.50 \mathrm{sq} \mathrm{km}$ area (14.57\%), $174.16 \mathrm{sq} \mathrm{km}$ area $(9.01 \%)$, and $52.49 \mathrm{sq} \mathrm{km}$ area (2.72\%) respectively. Bankura-Il block has very high and high adaptive capacity. Some part of Bankura-l is high and some part is shown moderate 
adaptive capacity. Santuri and some part of Gangajalghati have moderate adaptive capacity. Indpur, puncha, some portion of Hura, para and Saltora block has very low adaptive capacity. Raghunathpur-I, Kashipur, Chnatna, and some parts of Saltora also has low adaptive capacity.

Finally the Agricultural Drought Vulnerability Zone (ADVZ) map was prepared by using the ArcGIS environment. Combined three indices to assess the spatial distribution of agricultural drought vulnerability in the upper Dwarakeshwer river basin. Shown in Fig. 12.

There are five zones under the vulnerable category namely: very high ( $266.89 \mathrm{sq} \mathrm{km})$, high ( $547.05 \mathrm{sq} \mathrm{km})$, moderate (568.69 sq $\mathrm{km})$, low (186.68 sq km), and very low (362.32 sq km). South and southwestern part of the study area has been recognized as very high to high agricultural drought vulnerability, mainly Indpur, puncha, hura and para. Southeastern parts of Bankura-II block showing very low vulnerability. Some portion of Bankura-I and Santuri are noticed with low vulnerability. Remaining areas mainly Chhatna, Gangajolghati. Kasipur, Santuri and Raghunathpur have moderate to high drought vulnerability. Overall $13.82 \%$ of the study region is under very high vulnerability, $28.32 \%$ area of the study region is under high vulnerability. Moderate vulnerability affected portions cover almost $29.44 \%$ of the total area of the region and $9.66 \%$ area is under low vulnerability. The remaining portion ( $18.76 \%$ area) has very low agricultural drought vulnerability.

Higher adaptive capacity indicates lower vulnerability. Adaptive capacity can be controlled through environmental and sustainable socio-economic development. Sensitivity can be changed by changing the type of crop. But the exposure is associated with patterns of rainfall. So, it is not possible to control it. However, real time forecasting can be of some benefit.

\section{Validation}

There is no universally accepted, accurate, and direct mechanism has not developed for determining the validity of agricultural drought vulnerability (Murthy et al. 2015). Thus, some research papers have not been validated (Dalezios et al. 2012; Pei et al 2016; Kar et al. 2018). But many research papers have tried to determine the validity using some indirect methods such as comparative discussions with drought events of different years. In someresearch papers (He et al.2012) the empirical evidence of crop yield reduction with previous drought events (Zhao et al. 2011), crop yield variability of principal crop (Murthy et al 2015a), Yield Anomaly Index (YAI) (Dutta et al. 2015), Correlation of food grain productivity and HDI with drought events years (Sehgal and Dhakar 2016) are used to validate their respective work. Yield Anomaly Index (YAl) has been to determine the validation of this agricultural drought vulnerability map. YAl is a very reliable technology for determining the deviation of crop yield production in a particular year from long term mean. The following formula is used to calculate YAl:

$\mathrm{YAI}=(\mathrm{Y}-\mathrm{Xy}) / \mathrm{sd}$

Where,

Y - Crop yield, Xy - long term means yield, and sd - standard deviation.

Long term aman paddy yield data from 2004 to 2014 (Missing data in year 2009) has been used here. YAl of aman paddy of a drought year 2010 and a Normal (wet) year YAl of 2012 are used for comparative discussion (Fig. 13). It can be seen that almost every block has a negative YAl in the dry year of 2010 and a positive YAl in the normal wet year of 2012. The result shows that the entire region is a drought vulnerability region, which validates the prepared ADVZ.

\section{Discussion}

The above agricultural drought vulnerability analysis and mapping of the study were revealed for agricultural drought management purposes. This AHP and GIS-based unique methodology was used to reveal the $\mathrm{El}, \mathrm{SI}$, and $\mathrm{ACl}$ that determine the basin-scale drought vulnerability. Here, the principal exposure factor is rainfall that can determine the water scarcity due to precipitation deficit. Calculation of drought duration, intensity, and frequency of SPI over the 10 years' time period was done. On the other hand, satellite-based NDDI disclosed the drought condition of the study area. LULC is showing that the region is highly dependent on an agro-based economy. As a result, when drought phenomenon happens, the probability of potential losses of the agricultural sector in this basin is relatively very serious. The sensitivity of this area is mainly dependent on vegetation, soil moisture, and temperature. These are assessed by using remote sensing-based index meanly VHI, NDVI, EVI, NDVI, VCl, and TCl.

Page $17 / 33$ 
$\mathrm{TCl}$ materially monitors the surface temperature conditions. NDWI essentially monitors the soil moisture and vegetation index basically monitoring the greenness and health of crop conditions which can assess water stress on the crop over the whole seasons. The total adaptive capacity index demonstrates a region's capability to defend drought vulnerability.

The exposure index and the sensitivity index of region together increases the vulnerability to drought, while the adaptive capacity index builds the capacity of drought tolerance which reduces the drought vulnerability. The agricultural drought vulnerability map showed that Indpur, some part is puncha and para, northern part of Saltora, western part of Onda, middle portion of Chhatana, north western and middle parts of Kashipur block have high vulnerability for agricultural drought due to very low adaptive capacity. Western Hura, south western Kashipur, south eastern Saltora and Gangajalghati, eastern Onda, north eastern Chhatna block has moderate vulnerability for agricultural drought due to low sensitivity, low adaptive capacity, high to moderate exposure index. Bankura-I and Santuri block has under the low vulnerability zone because moderate to high adaptive capacity and moderate to low sensitivity index. Bankura - II block has very low vulnerability due to very high adaptive capacity and low sensitivity index. So assessment of agricultural drought vulnerability adaptive capacity is a principal controlling factor that regulating to decrease the agricultural drought vulnerability. The irrigation area density, rural education and ground water depth, also drainage and soil condition, agricultural labour density, crop production density, agricultural are density are the important regional parameter for agricultural drought vulnerability assessment. According to regional nature of upper Dwarakeshwar river basin that region is under highly agricultural vulnerable when drought occurs. So, 1) To minimization of the ratio of agricultural dependent population, 2) spreading of economic diversification, 3) promotion of drought resistant crop farming and 4) increase investment for replace the traditional irrigation system to modern (sprikel, drip, pipe, infiltration) irrigation system are the main way to reduce the ADV. That is why; diagnostic assessment of three indices-based agricultural drought vulnerability map of agrobased economic region is required for a drought reduction plan.

\section{Conclusion}

Drought is a major hidden catastrophe of agricultural production in any region of the world. As a result, due to climate change, agricultural drought risk management is very necessary for maintaining food security. The current study has accepted a three dimensional holistic perception for assessment and spatial distribution of agricultural drought vulnerability map. Agricultural drought vulnerability considers address the multidimensional nature of multiple parameters such as exposure factors like LULC,NDDI and daily rainfall based 3-month SPI(drought duration, intensity, frequency), sensitive factor like NDVI, NDWI, VCl, TCl, $\mathrm{VHI}, \mathrm{EVI}$ and adaptive capacity factors like Economic, social and environmental adaptive capacity. Different input indicators were selected by studying the various vulnerabilities related to climate and these inputs are managed in a systematic way to diagnose the spatial distribution of agricultural drought vulnerability. Weights were selected based on the ability of the parameters involved in the subjectivity to affect that particular index. Finally, an agricultural drought vulnerability map has been created using those three indices. Ultimatly vulnerability maps will assist in drought management, identifying agricultural areas affected by extreme drought. This will greatly benefit the planners and government officials in formulating government policy for local scale drought management strategy. It also demonstrates the effectiveness of remote sensing and GIS-based three-dimensional methodology for identifying drought-related stresses in the agricultural economy. Thus, by modifying or directly using this methodology, it is possible to assess the agricultural drought in any part of the world and to formulate management policies based on it.

\section{Abbreviations}

NDDI, Normalized Difference Drought Index; LULC, Landuse and Landcover; ADI, Average Drought Intensity; DF, Drought Frequency, ADD, Average Drought Duration; PI, Peak Intensity; VHI, Vegetation Health Index; NDWI, Normalized Difference Water Index; EVI, Enhanced Vegetation Index; NDVI, Normalized Difference Vegetation Index; VCl, Vegetation Condition Index; LST, Land Surface Temperature, TCl, Temperature Condition Index;

\section{Declarations}

Acknowledgments 
The authors are grateful to University Grant Commission (UGC), New Delhi for providing Junior Research Fellowship [UGC-Ref. No: 3272/(NET-JULY 2016)] in favor of first author for conducting the research work. They are also thankful to United States Geological Survey (USGS), Google Earth Engine (GEE), National Centers for Environmental Prediction (NCEP), NBSS \& LUP, IMD Pune, CGWB.

Author contribution

Conceptualization, Methodology development, Formal analysis and investigation: Ujjal Senapati. Writing-original draft preparation and review and editing: Ujjal Senapati and Tapan Kumar Das.

Funding

This study was funded by the University Grant Commission (UGC), New Delhi for providing Junior Research Fellowship [UGC-Ref. No: 3272/(NET-JULY 2016)]

Data availability

All the data used for this study are available and provided by the public entities. Data sources are given in Table 1.

Code availability: Not applicable.

Ethics approval: Not applicable.

Consent to participate: Not applicable.

Consent for publication: Not applicable.

Conflict of interest: The authors declare no competing interests

\section{References}

1. Amalo LF, Ma'rufah U, Permatasari PA (2018) Monitoring 2015 drought in West Java using Normalized Difference Water Index (NDWI). IOP Conference Series: Earth and Environmental Science, 149, 012007. https://doi:10.1088/17551315/149/1/012007

2. Arekhi M, Saglam S, Ozkan UY (2019) Drought monitoring and assessment using Landsat TM/OLI data in the agricultural lands of Bandar-e-Turkmen and Gomishan cities, Iran. Environ

3. Badamassi MBM, Ahmed EA, Gbetkom PG (2019) A New Agricultural Drought Index to Better Detect and Monitor Millet Crop by Remote Sensing in West Africa: Case of Niger. J Adv Res Dyn Control Syst 11(11-Special Issue):10231033.https://doi:10.5373/JARDCS/V11SP11/20193132 https://hal.archives-ouvertes.fr/hal-02508275/document

4. Bates BC, Kundzewicz ZW, Wu S, Palutikof JP (2008) Climate Change and Water: Intergovernmental Panel on Climate Change (IPCC) Technical Paper VI. IPCC Secretariat, Geneva

5. Bera A, Mukhopadhyay BP, Das D (2019) Landslide hazard zonation mapping usingmulti-criteria analysis with the help of GIS techniques: a case study from eastern Himalayas, Namchi, South Sikkim. Nat Hazards 96:935-959.

https://doi.org/10.1007/s11069-019-03580-w

6. Bhavani P, Chakravarthi V, Roy PS, Joshi PK, Chandrasekar K (2017) Long-term agricultural performance and climate variability for drought assessment: a regional study from Telangana and Andhra Pradesh states, India. Geomatics. Nat Hazards Risk 8(2):822-840. https://doi:10.1080/19475705.2016.1271831

7. Bhunia P, Das P, Maiti R (2020) Meteorological drought study through SPI in three drought prone districts of west Bengal, India. Earth Syst Environ 4(1):43-55. https://doi.org/10.1007/s41748-019-00137-6

8. Biazin B, Sterk G (2013) Drought vulnerability drives land-use and land cover changes in the Rift Valley dry lands of Ethiopia. Agric Ecosyst Environ 164:100-113. https://doi:10.1016/j.agee.2012.09.012 
9. Boken VK, Cracknell AP, Heathcote RL (2005) Monitoring and predicting agricultural drought: a global study. Oxford University Press

10. Chockalingam J, Giriraj A, Avishek K, Mondal S (2015) AGRICULTURE DROUGHT ASSESSMENT AND MONITORING (ADAMS) SOFTWARE USING ESRI ArcMap.https://www.researchgate.net/publication/284170143

11. Dalezios NR, Blanta A, Spyropoulos NV (2012) Assessment of remotely sensed drought features in vulnerable agriculture. Nat Hazards Earth Syst Sci 12(10):3139-3150. https://doi:10.5194/nhess-12-3139-2012

12. Dembele M, SJ Zwart (2016) An assessment of agricultural drought events in Burkina Faso between 2001 and 2014 - a spatially explicit analysis using remotely sensed data of vegetation, surface temperature and precipitation. AfricaRice GIS Report - 3. Africa Rice Center, Cotonou, Benin Dev Sustain.https://doi:10.1007/s10668-019-00509-y

13. Dutta D, Kundu A, Patel NR (2013) Predicting agricultural drought in eastern Rajasthan of India using NDVI and standardized precipitation index. Geocarto Int 28(3):192-209. https://doi.org/10.1080/10106049.2012.679975

14. Dutta D, Kundu A, Patel NR, Saha SK, Siddiqui AR (2015) Assessment of agricultural drought in Rajasthan (India) using remote sensing derived Vegetation Condition Index (VCI) and Standardized Precipitation Index (SPI). Egypt J Remote Sens Sp Sci 18(1):53-63. https://doi.org/10.1016/j.ejrs.2015.03.006

15. Ekrami M, Marj AF, Barkhordari J, Dashtakian K (2016) Drought vulnerability mapping using AHP method in arid and semiarid areas: a case study for Taft Township, Yazd Province, Iran. Environ Earth Sci 75(12):1039. https://doi:10.1007/s12665-016-5822-z

16. Fischer AP, Frazier TG (2017) Social Vulnerability to Climate Change in Temperate Forest Areas: New Measures of Exposure, Sensitivity, and Adaptive Capacity. Ann Am Assoc Geogr 108(3):658-678. https://doi:10.1080/24694452.2017.1387046

17. Ghosh KG (2019) Spatial and temporal appraisal of drought jeopardy over the Gangetic West Bengal, eastern India. Geoenviron Disasters. https://doi.org/10.1186/s4067 7-018-0117-1

18. Gu Y, Brown JF, Verdin JP, Wardlow B (2007) A five-year analysis of MODIS NDVI and NDWI for grassland drought assessment over the central Great Plains of the United States. Geophys Res Lett 34(6). https://doi:10.1029/2006gl029127

19. He B, Wu J, Lü A, Cui X, Zhou L, Liu M, Zhao L (2012) Quantitative assessment and spatial characteristic analysis of agricultural drought risk in China. Nat Hazards 66(2):155-166. https://doi:10.1007/s11069-012-0398-8

20. Hundera H, Berhan G, Bewuket W (2016) Remote sensing and GIS based agricultural drought risk assessment in east Shewa zone, central Rift Valley region of Ethiopia J Environ Earth Sci 6(7)

21. Jain VK, Pandey RP, Jain MK (2014) Spatio-temporal assessment of vulnerability to drought. Nat Hazards 76(1):443-469. https://doi:10.1007/s11069-014-1502-z

22. Kar SK, Thomas T, Singh RM, Patel L (2018) Integrated assessment of drought vulnerability using indicators for Dhasan basin in Bundelkhand region, Madhya Pradesh, India. Curr Sci 115(2):338-346

23. Kar SK, Thomas T, Singh RM, Patel L (2018) Integrated assessment of drought vulnerability using indicators for Dhasan basin in Bundelkhand region, Madhya Pradesh, India. Curr Sci 115(2):338-346. doi 10.18520/cs/v115/i2/338-346

24. Kim SM, Kang MS, Jang MW (2018) Assessment of agricultural drought vulnerability to climate change at a municipal level in South Korea. Paddy Water Environ 16:699-714. https://doi.org/10.1007/s10333-018-0661-z

25. Kumar A, Krishna AP (2018) Assessment off groundwater potential zones in coal mining impacted hard-rock terrain of India by integrating geospatial and analytic hierarchy process (AHP) approach. Geocarto Int 33(2):105-129.

https://doi:10.1080/10106049.2016.1232314

26. Murthy CS, Laxman B, Sesha Sai MVRS (2015a) Geospatial analysis of agricultural drought vulnerability using a composite index based on exposure, sensitivity and adaptive capacity. Int J Disaster Risk Reduct 12:163-171.

https://doi.org/10.1016/j.ijdrr.2015.01.004

27. Murthy CS, Sesha Sai MVR, Das PK, Chakraborty NKM, Dwivedi A RS (2010) Assessing agricultural drought vulnerability using time series rainfall and NDVI. NATIONAL NATURAL RESOURCES MANAGEMENT SYSTEM. https://citeseerx.ist.psu.edu/viewdoc/download?doi=10.1.1.461.1682\&rep=rep1\&type=pdf\#page=70

28. Murthy CS, Yadav M, Ahamed JM, Laxman B, Prawasi R, Sai MS, Hooda RS (2015b) A study on agricultural drought vulnerability at disaggregated level in a highly irrigated and intensely cropped state of India. Environ Monit Assess 
187(3):140. https://doi.org/10.1007/s10661-015-4296-x

29. Nagarajan R (2009) Drought assessment. Springer Science \& Business Media. ISBN: 81-85589-78-x

30. Palchaudhuri M, Biswas S (2019) Application of LISS III and MODIS-derived vegetation indices for assessment of micro-level agricultural drought. Egypt J Remote Sensing Space Sci. https://doi:10.1016/j.ejrs.2019.12.004

31. Pandey S, PandeyAC, Nathawat MS, Kumar M, Mahanti NC (2012) Drought hazard assessment using geoinformatics over parts of Chotanagpur plateau region, Jharkhand, India. Nat Hazards 63(2):279-303. https://doi:10.1007/s11069-012-0093-9

32. Park H, Kim K, Lee DK (2019) Prediction of Severe Drought Area Based on Random Forest: Using Satellite Image and Topography Data. Water 11(4):705. https://doi:10.3390/w11040705

33. Pei W, Fu Q, Liu D, Li TX, Cheng K (2016) Assessing agricultural drought vulnerability in the Sanjiang Plain based on an improved projection pursuit model. Nat Hazards 82(1):683-701. https://doi:10.1007/s11069-016-2213-4

34. Prabnakorn S, Maskey S, Suryadi FX, de Fraiture C (2019) Assessment of drought hazard, exposure, vulnerability, and risk for rice cultivation in the Mun River Basin in Thailand. Nat Hazards. https://doi:10.1007/s11069-019-03681-6

35. Raha S, Gayen SK (2020) Simulation of meteorological drought using exponential smoothing models: a study on Bankura District, West Bengal, India. SN Appl Sci 2(5). https://doi:10.1007/s42452-020-2730-3

36. Rahman MR, Lateh H (2016) Meteorological drought in Bangladesh: assessing, analysing and hazard mapping using SPI, GIS and monthly rainfall data. Environ Earth Sci 75(12):1026. https://doi:10.1007/s12665-016-5829-5

37. Ramadas M, Govindaraju RS (2015) Probabilistic assessment of agricultural droughts using graphical models. J Hydrol 526:151-163. https://doi:10.1016/j.jhydrol.2014.09.026

38. Rojas O, Vrieling A, Rembold F (2011) Assessing drought probability for agricultural areas in Africa with coarse resolution remote sensing imagery. Remote Sens Environ 115(2):343-352. https://doi:10.1016/j.rse.2010.09.006

39. Saaty TL (1980) The analytic hierarchy process. McGraw-Hill, New York

40. Saaty TL (1990) How to make a decision: the analytic hierarchy process. Eur J Oper Res 48:9-26. https://doi.org/10.1016/0377-2217(90)90057-I

41. Sanchez N, Gonzalez-Zamora A, Martinez-Fernandez J, Piles M, Pablos M (2018) Integrated remote sensing approach to global agricultural drought monitoring. Agric For Meteorol 259:141-153. https://doi:10.1016/j.agrformet.2018.04.022

42. Sehgal VK, Dhakar R (2016) Geospatial approach for assessment of bioEcomonic vulnerability to agricultural drought and its intra-seasonal variations. Environ Monit Assess 188(3):197. https://doi:10.1007/s10661-016-5187-5

43. Sener S, Sener E, Karagüzel R (2011) Solid waste disposal site selection with GIS and AHP methodology: a case study in Senirkent-Uluborlu (Isparta) Basin, Turkey. Environ Monit Assess 173(1-4):533-554. https://doi:10.1007/s10661-010-1403$\mathrm{X}$

44. Sun H, Zhao X, Chen Y, Gong A, Yang J (2013) A new agricultural drought monitoring index combining MODIS NDWI and day-night land surface temperatures: a case study in China. Int J Remote Sens 34(24):8986-9001. https://doi:10.1080/01431161.2013.860659

45. Trnka M, Hlavinka P, Mozny M, Semeradova D, Stepanek P, Balek J, Bartosova L, Zahradnícek P, Blahova M, Skalak P, Farda A, Hayes M, Svoboda M, Wagner W, Eitzinger J, Fischer M, Zalud Z (2020) Czech Drought Monitor System for Monitoring and Forecasting Agricultural Drought and Drought Impacts. Int J Climatol. https://doi:10.1002/joc.6557

46. Wang Z, He F, Fang W, Liao Y (2013) Assessment of Ecomonic vulnerability to agricultural drought in China. Nat Hazards 67(2):645-657. https://doi:10.1007/s11069-013-0594-1

47. Weis SWM, Agostini VN, Roth LM, Gilmer B, Schill SR, Knowles JE, Blyther R (2016) Assessing vulnerability: an integrated approach for mapping adaptive capacity, sensitivity, and exposure. Clim Change 136(3-4):615-629.

https://doi:10.1007/s10584-016-1642-0

48. Wijitkosum S (2018) Fuzzy AHP for drought risk assessment in Lam Ta Kong watershed, the north-eastern region of Thailand. Soil Water Res 13:218-225. https://doi.org/10.17221/158/2017-SWR

49. Wilhite DA (2000) Drought as a natural hazard: concepts and definitions. In: Wilhite DA(ed.). Drought: A Global Assessment, Natural Hazards and Disasters Series. Routledge Publishers, London, pp 3-18

Page 21/33 
50. Zeng Z, Wu W, Li Z, Zhou Y, Guo Y, Huang H (2019) Agricultural Drought Risk Assessment in Southwest China. Water 11(5):1064. https://doi:10.3390/w11051064

51. Zhao H, Ge G, Xiaodong Y, Qiang Z, Meiting H, Yeyu Z, Zhan T (2011) Risk assessment of agricultural drought using CERESwheat model: a case study of Henan Plain, China. Clim Res 50:247-256. https://doi:10.3354/ cr01060

52. Zhu Z, Fu Y, Woodcock CE, Olofsson P, Vogelmann JE, Holden C, Wang M, Dai S, Yu Y (2016) Including land cover change in analysis of greenness trends using all available Landsat 5, 7, and 8 images: A case study from Guangzhou, China (20002014). Remote Sens Environ 185:243-257. https://doi:10.1016/j.rse.2016.03.036

\section{Figures}
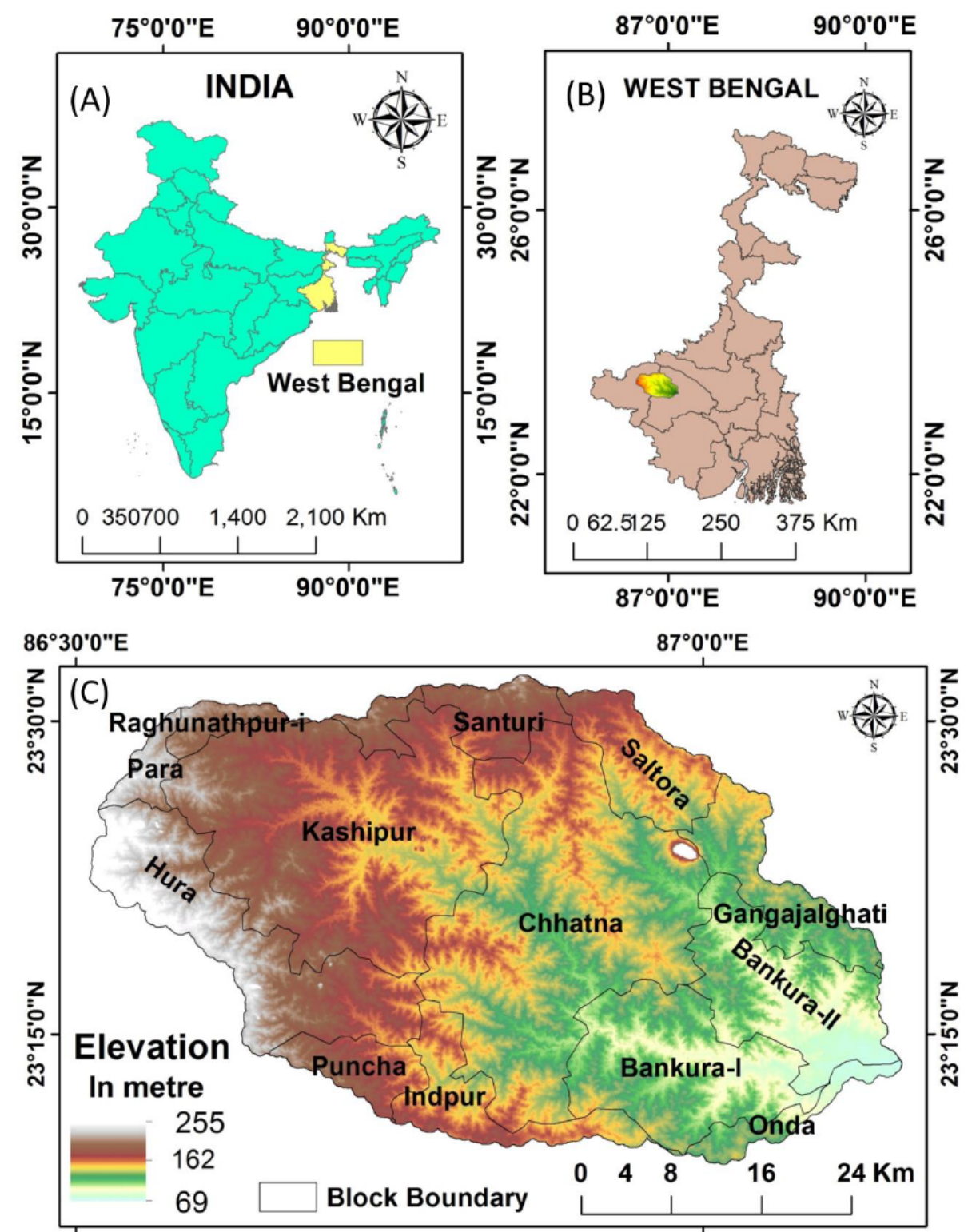

$86^{\circ} 30^{\circ} 0^{\prime \prime} \mathrm{E}$

$87^{\circ} 0^{\circ} 0 " \mathrm{E}$

Figure 1

Location map of the study area: (A) India, (B) West Bengal, and (C) Spatial distribution of associated blocks of upper Dwarakeshwar river basin. 


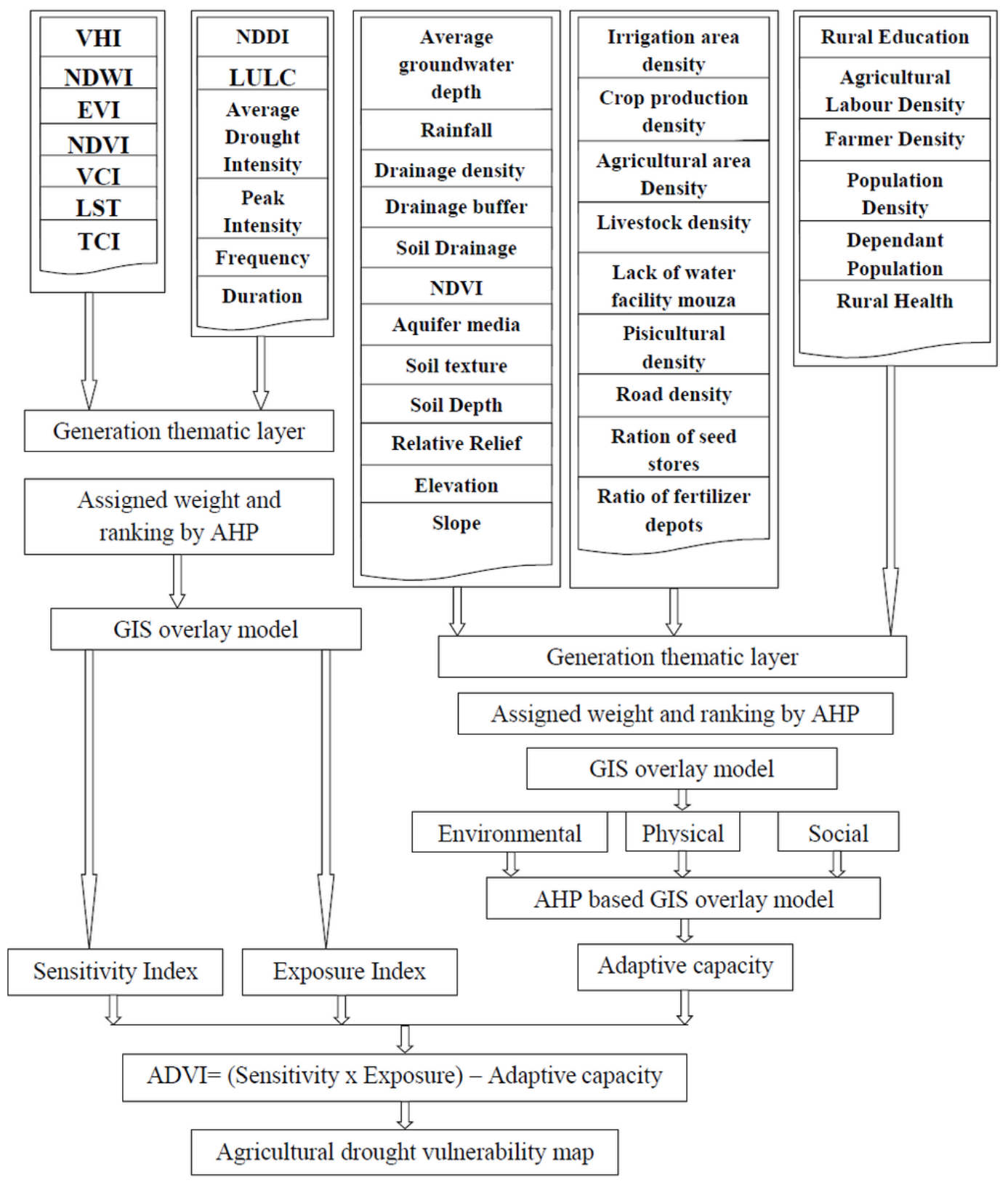

Figure 2

Flowchart of Agricultural drought vulnerability index Zone map methodology. 

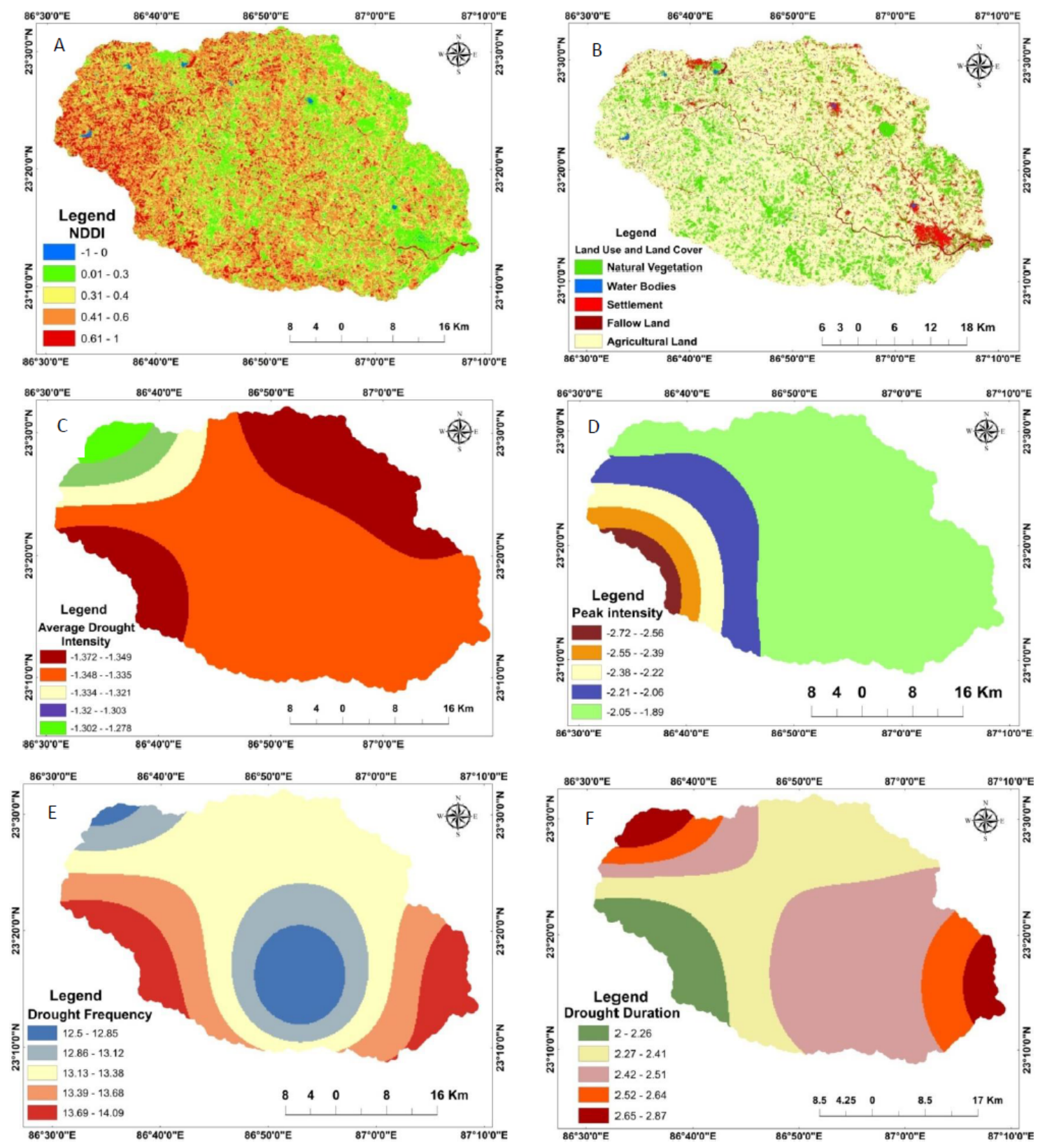

\section{Figure 3}

Thematic layers for Exposure index (A) NDDI, (B) Land use and Land cover, (C) Average Drought Intensity, (D) Peak Intensity, (E) Drought Frequency, (F) Drought Duration. 

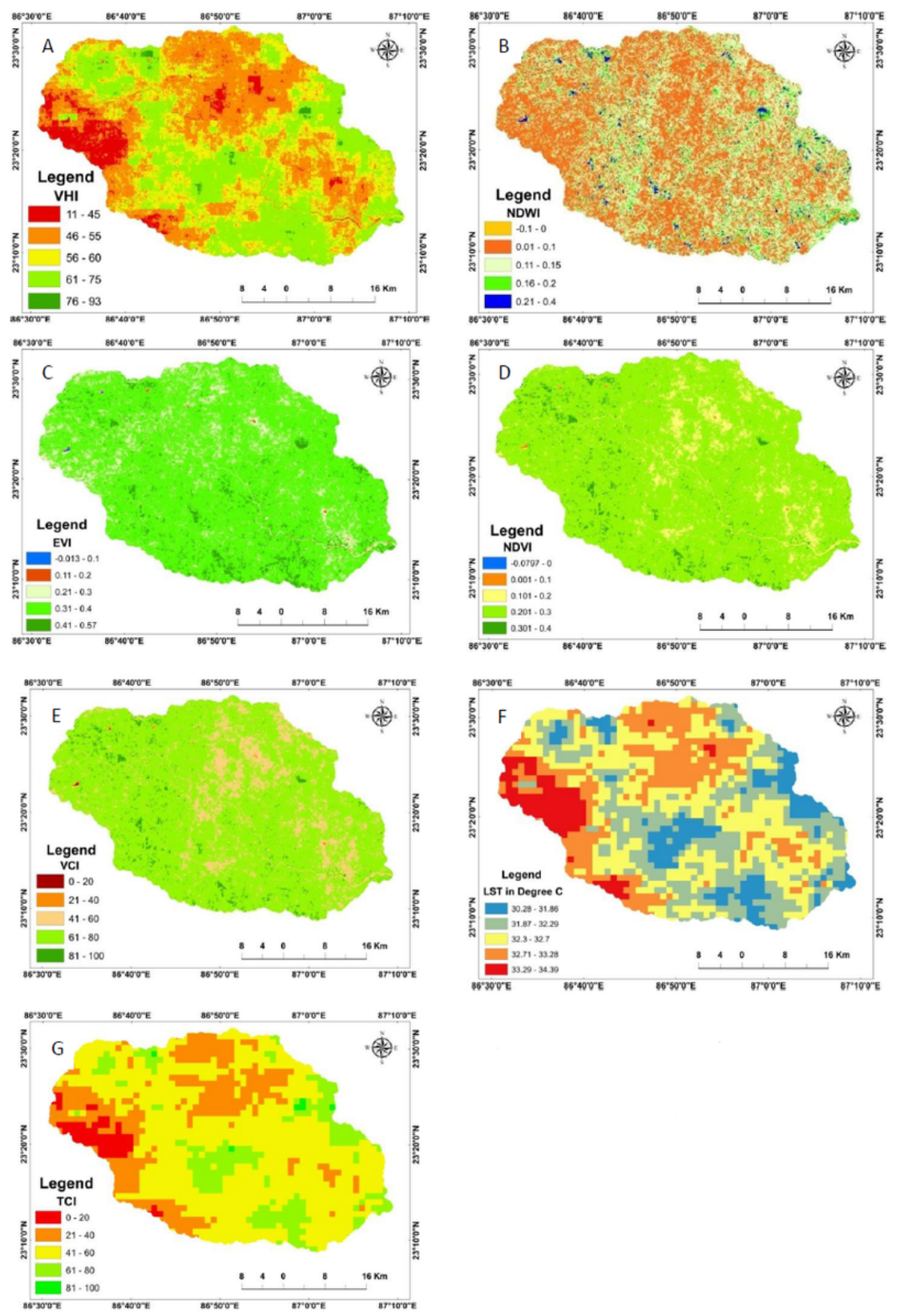

\section{Figure 4}

Thematic layers for Sensitivity index (A) VHI, (B) NDWI, (C) EVI, (D) NDVI (E) VCl, (F) LST, (G) TCl 

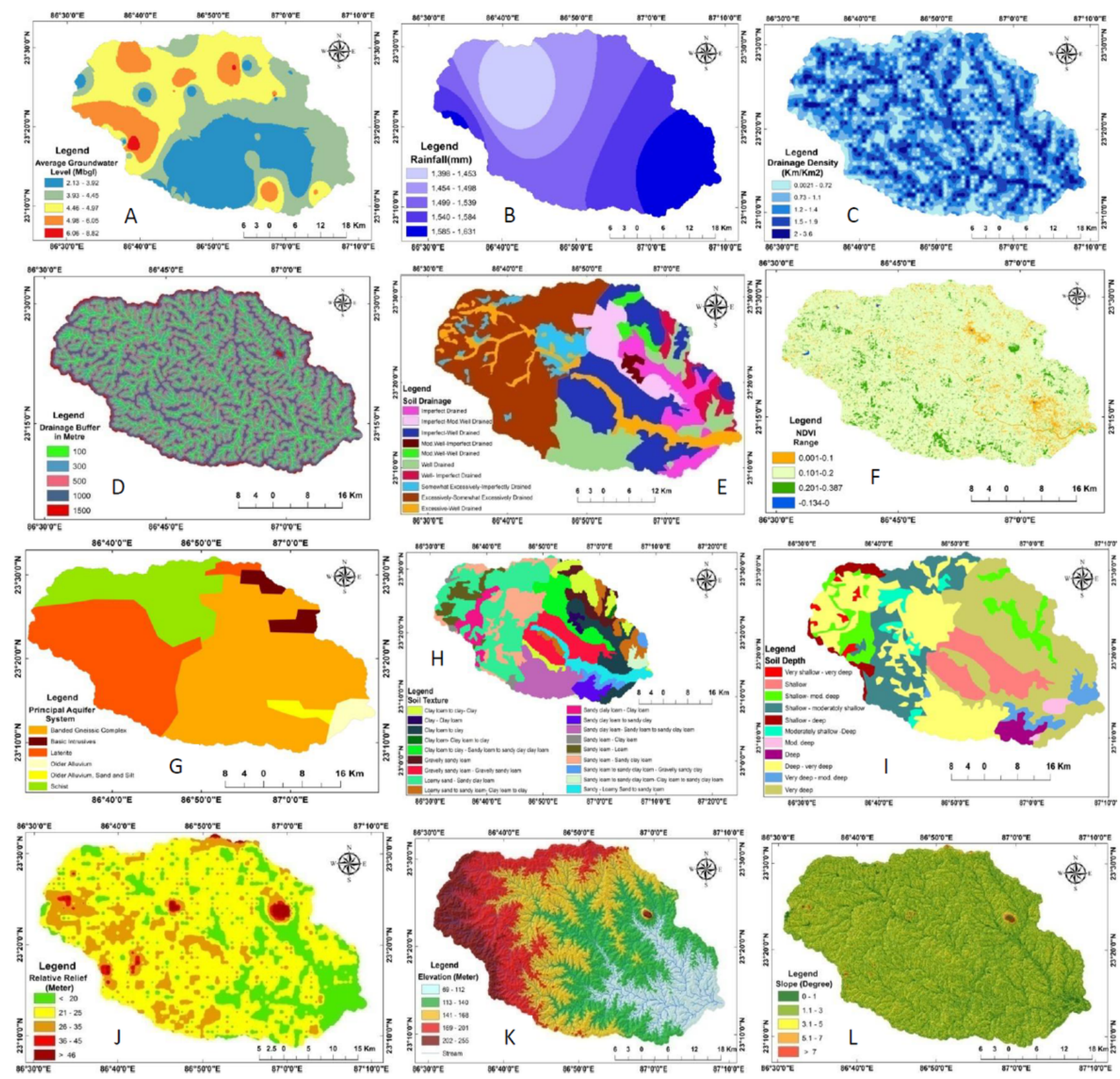

\section{Figure 5}

Thematic layers for Environmental Adaptive capacity (A) Average groundwater depths, (B) Rainfall, (C) Drainage density, (D) Drainage buffer, (E) Soil drainage, (F) NDVI, (G) Principal aquifer system, (H) Soil texture, (I) Soil depth, (J) Relative relief, (K) Elevation, (L) Slope. 

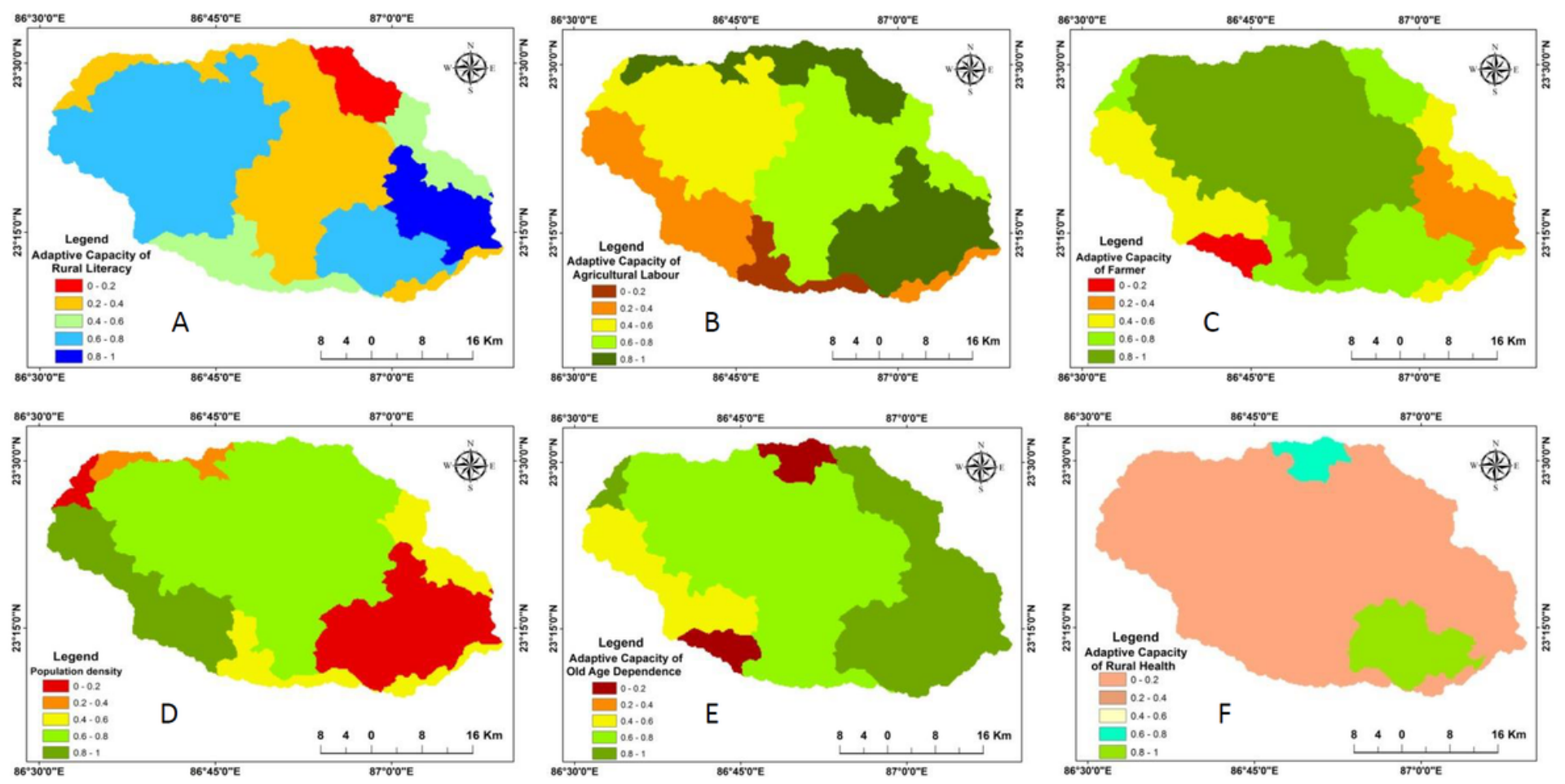

\section{Figure 6}

Thematic layers for Social Adaptive capacity (A) Rural literacy, (B) Agricultural Labour, (C) Farmer, (D) Population density, (E) Old age dependence, (F) Rural Health. 

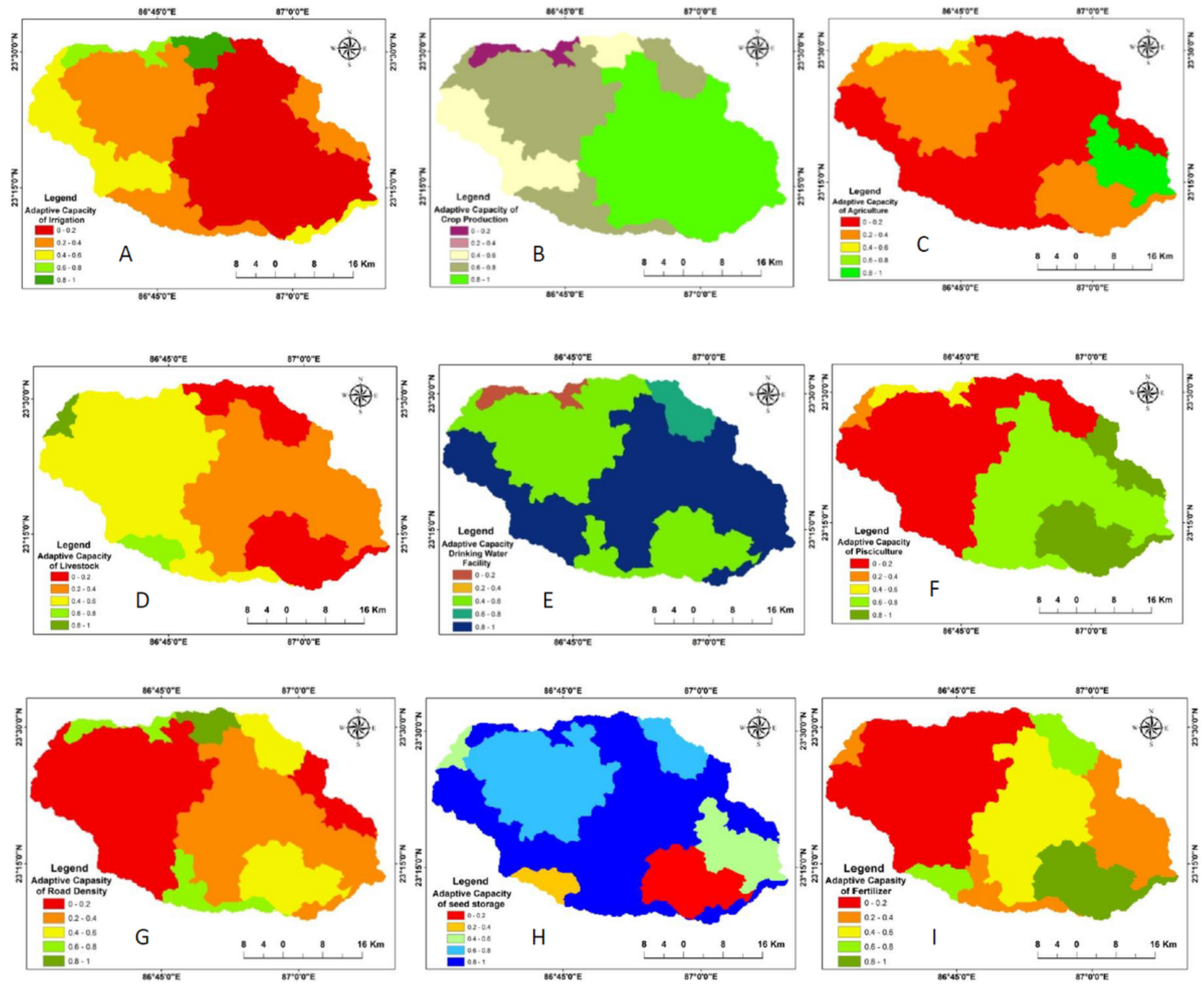

\section{Figure 7}

Thematic layers for Physical Adaptive capacity (A) Irrigation, (B) Crop production, (C) Agriculture, (D) Livestock, (E) Drinking water facility, (F) Pisciculture, (G) Road density, (H) Seed storage, (I) Fertilizer. 


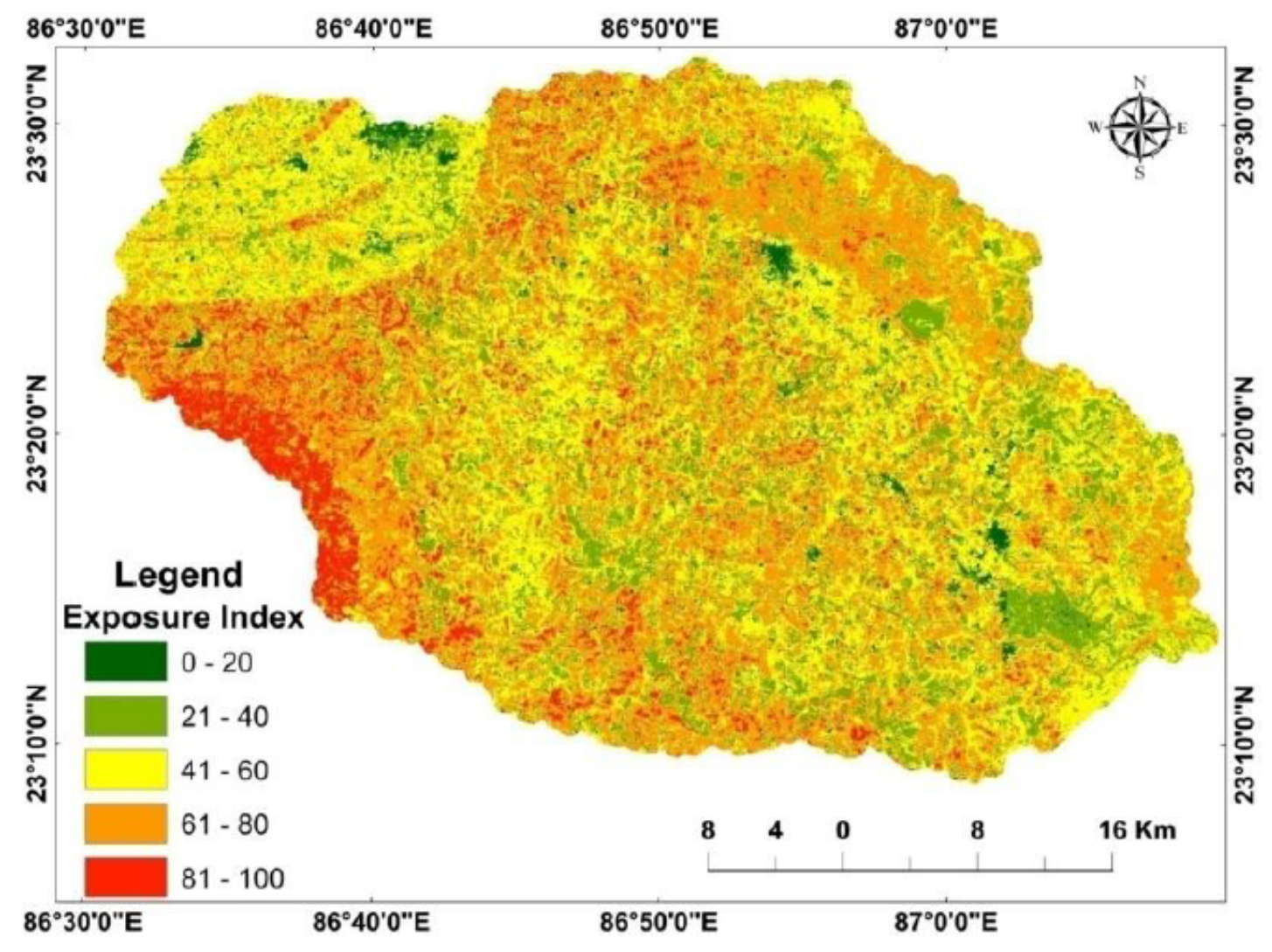

Figure 8

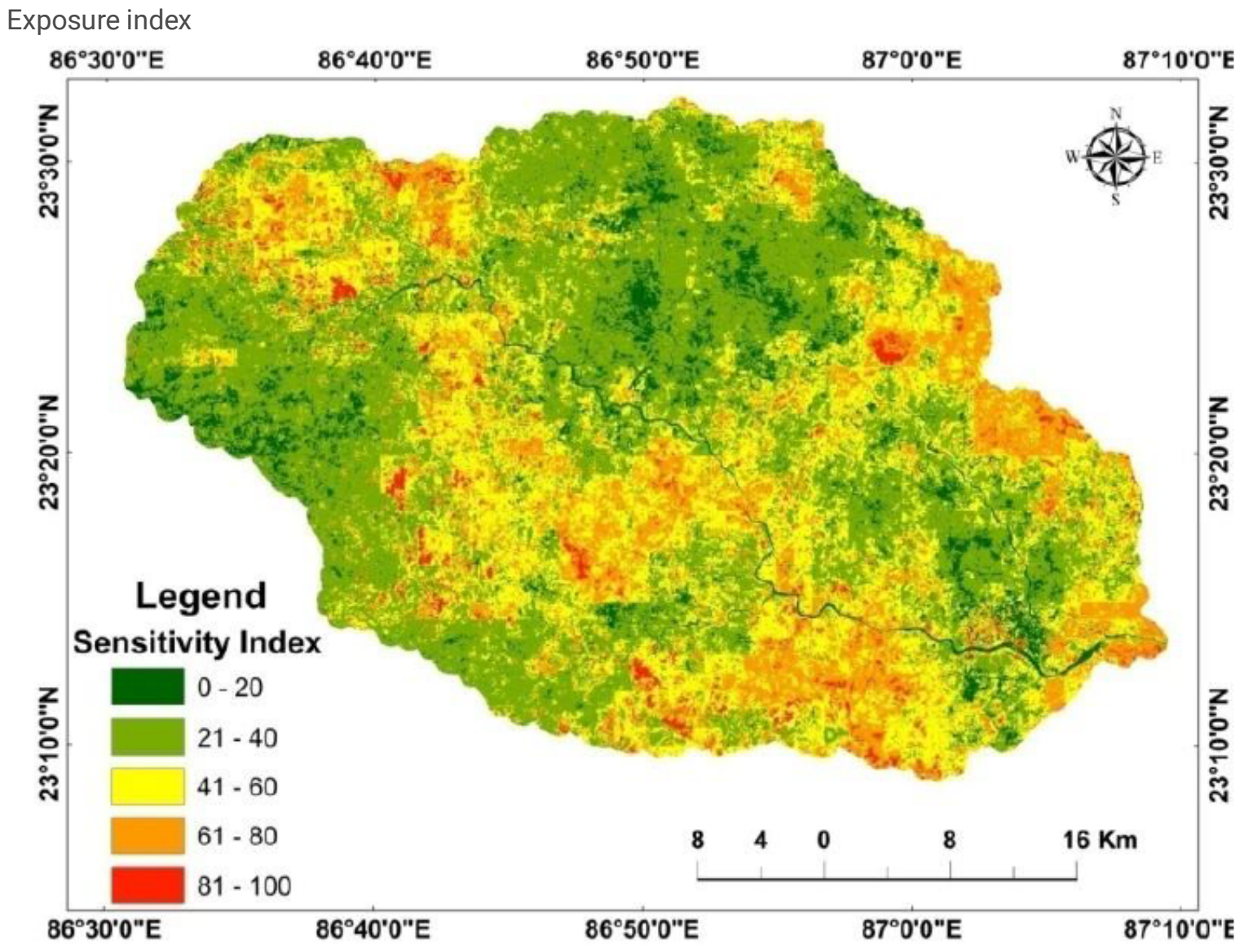

Figure 9 
Sensitivity index

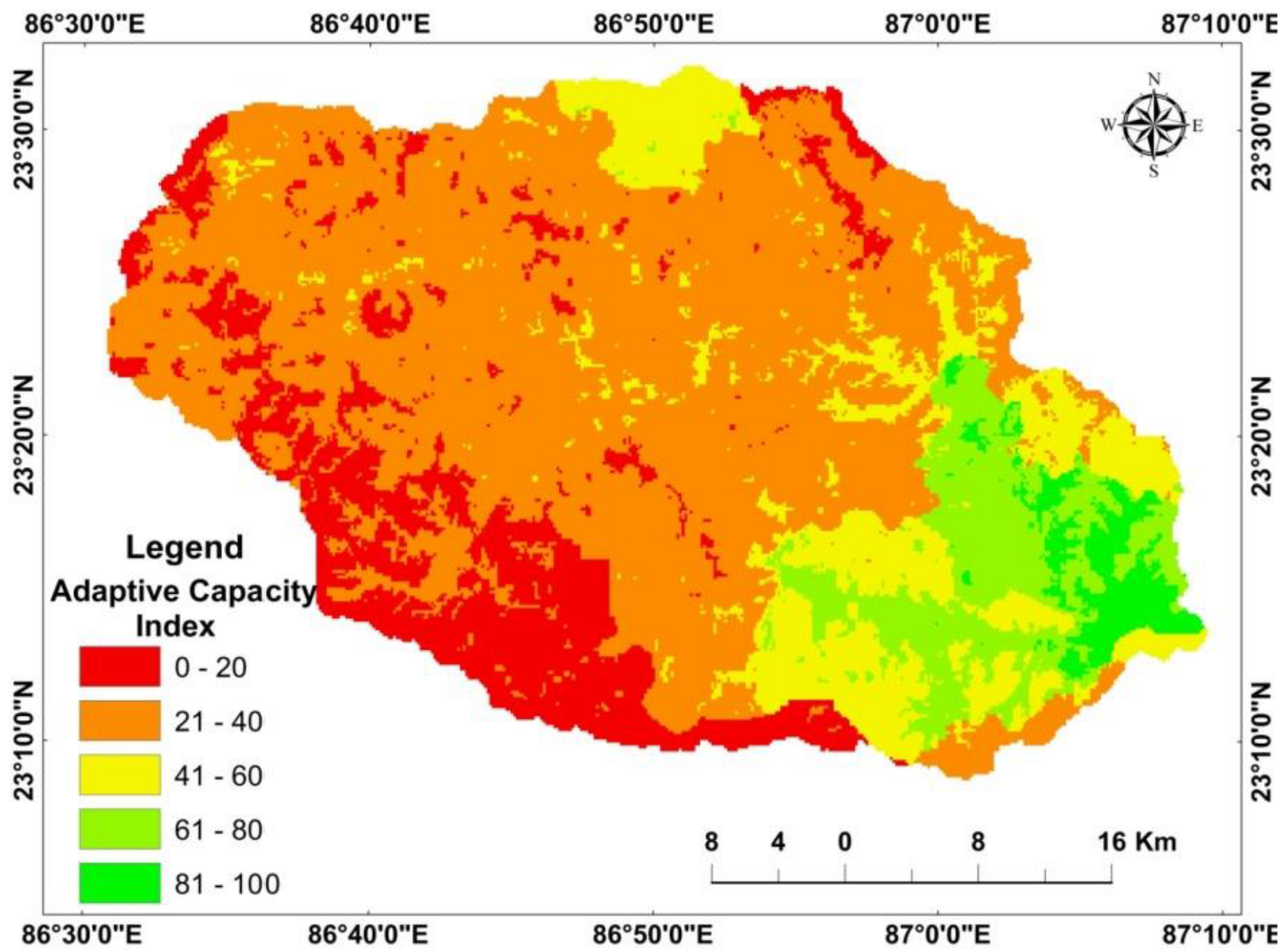

Figure 10

Adaptive capacity index 

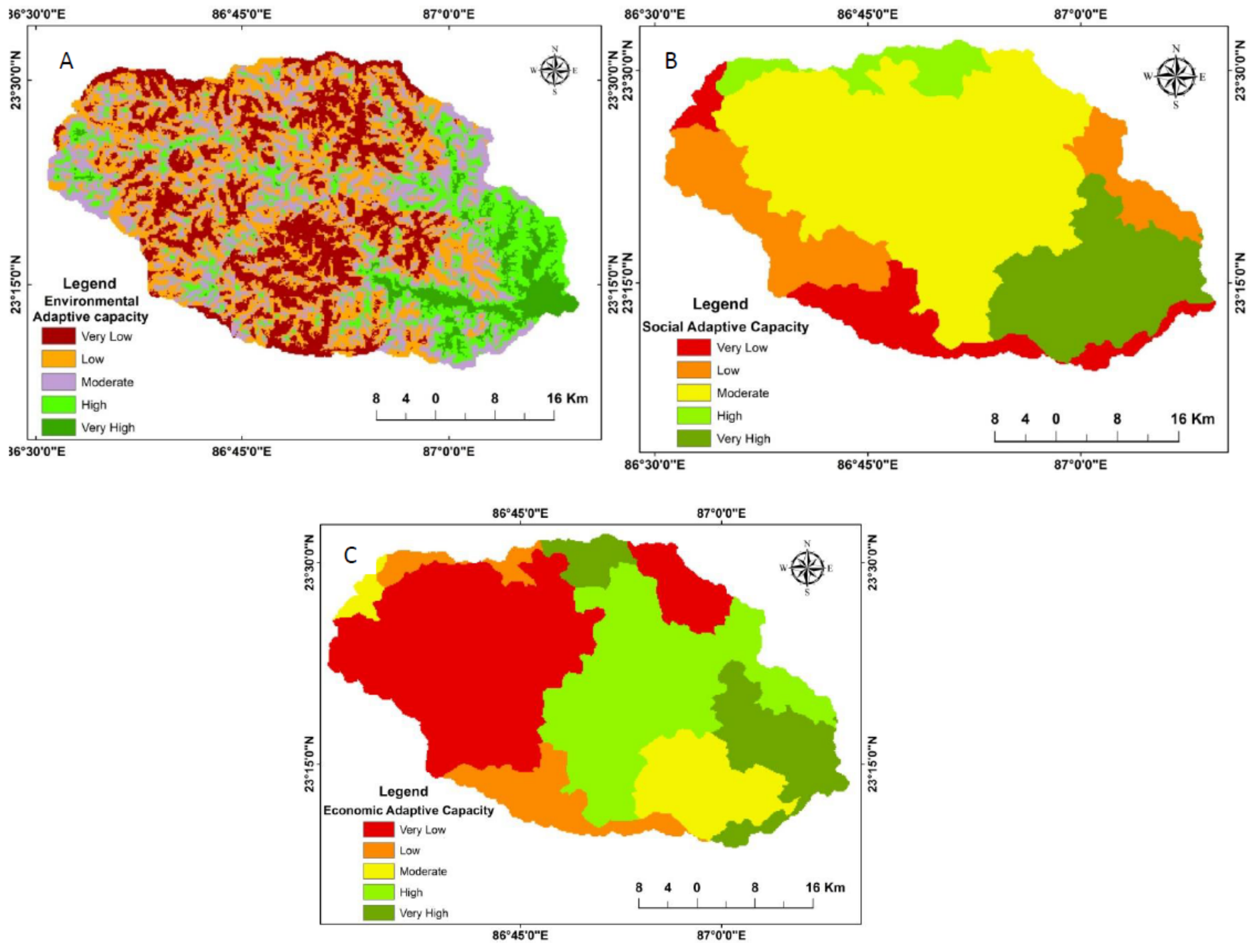

Figure 11

(A) Social adaptive capacity, (B) Environmental adaptive capacity, and (C) Economic adaptive capacity. 


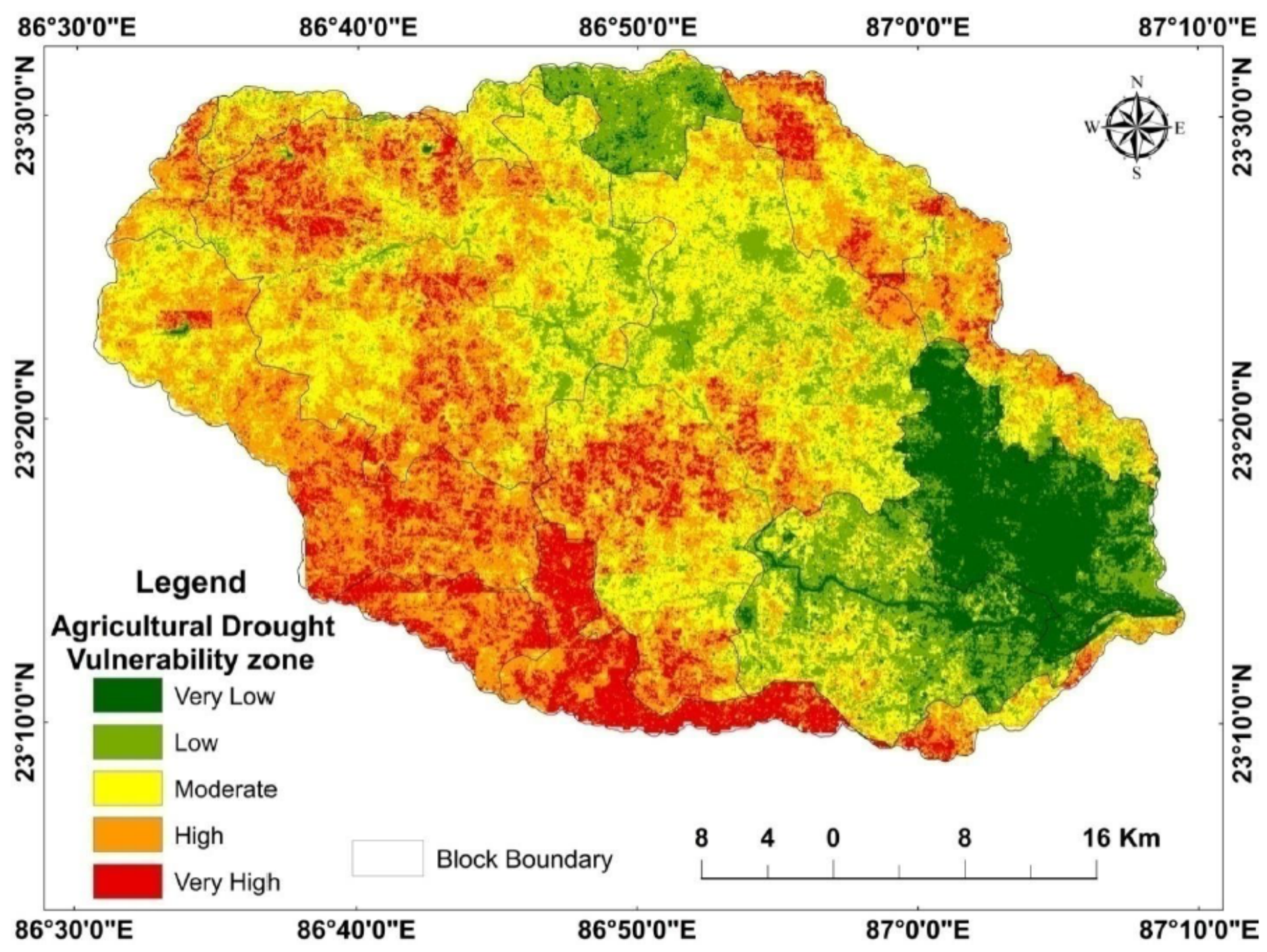

Figure 12

Agricultural drought vulnerability zones. 


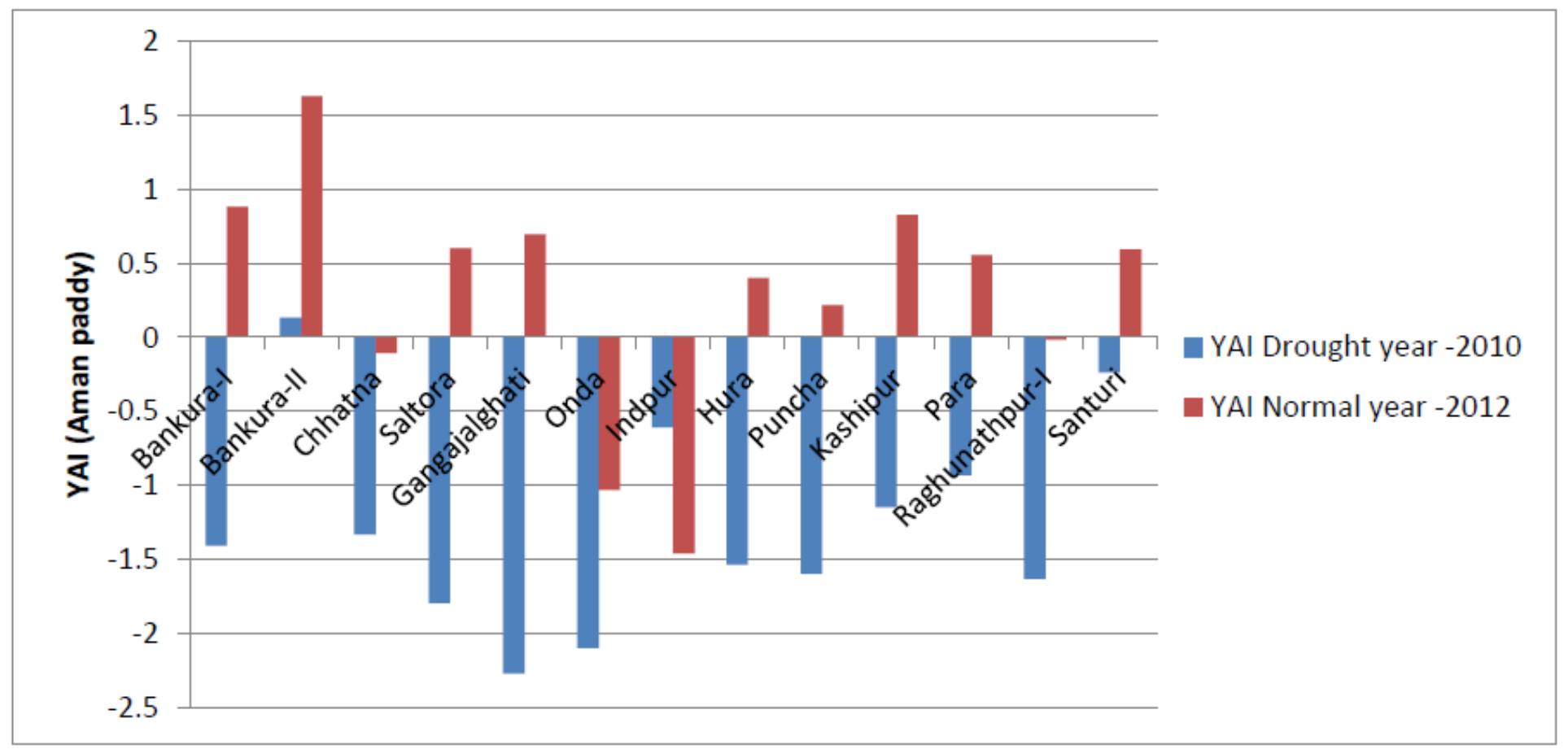

\section{Figure 13}

Block wise Yield anomaly index (YAl) of drought year (2010) and normal year (2012). 